ARTICLE

Received 23 Mar 2015 | Accepted 10 Apr 2015 | Published 13 May 2015

\title{
Directional cell movement through tissues is controlled by exosome secretion
}

Bong Hwan Sung ${ }^{1}$, Tatiana Ketova ${ }^{2}$, Daisuke Hoshino ${ }^{3}$, Andries Zijlstra ${ }^{1,2, \star}$ \& Alissa M. Weaver ${ }^{1,2,4, \star}$

Directional cell movement through tissues is critical for multiple biological processes and requires maintenance of polarity in the face of complex environmental cues. Here we use intravital imaging to demonstrate that secretion of exosomes from late endosomes is required for directionally persistent and efficient in vivo movement of cancer cells. Inhibiting exosome secretion or biogenesis leads to defective tumour cell migration associated with increased formation of unstable protrusions and excessive directional switching. In vitro rescue experiments with purified exosomes and matrix coating identify adhesion assembly as a critical exosome function that promotes efficient cell motility. Live-cell imaging reveals that exosome secretion directly precedes and promotes adhesion assembly. Fibronectin is found to be a critical motility-promoting cargo whose sorting into exosomes depends on binding to integrins. We propose that autocrine secretion of exosomes powerfully promotes directionally persistent and effective cell motility by reinforcing otherwise transient polarization states and promoting adhesion assembly.

\footnotetext{
${ }^{1}$ Department of Cancer Biology, Vanderbilt University Medical Center, Nashville, Tennessee 37232, USA. ${ }^{2}$ Department of Pathology, Microbiology and Immunology, Vanderbilt University Medical Center, Nashville, Tennessee 37232, USA. ${ }^{3}$ Division of Cancer Cell Research, Kanagawa Cancer Center, Yokohama 241-8515, Japan. ${ }^{4}$ Department of Cell and Developmental Biology, Vanderbilt University Medical Center, Nashville, Tennessee 37232, USA. * These authors contributed equally to this work. Correspondence and requests for materials should be addressed to A.Z. (email: andries.zijlstra@vanderbilt.edu) or to A.M.W. (email: alissa.weaver@vanderbilt.edu).
} 
$\mathrm{D}$ irectional cell movement involves the coordination of multiple subcellular processes. In order for cells to migrate effectively, polarization signals and protrusions must be stabilized to form a dominant leading-edge protrusion ${ }^{1}$. Adhesion assembly is likely to significantly contribute to signal reinforcement at the leading edge ${ }^{\text {; }}$ however, the mechanisms by which cells spatially coordinate adhesion assembly during persistent cell migration are poorly understood. In tissues, the extracellular matrix (ECM) environment adds further complexity that could provide conflicting signals to migrating cells if it is not coordinated with chemical gradients.

One potential mechanism that could coordinate initial directional signalling with adhesion formation is vesicle trafficking. Polarization of and trafficking from the Golgi apparatus has been shown to regulate cell migration, although the cargoes are unknown ${ }^{2-4}$. Endocytic recycling of integrins has also been shown to impact cell motility, ${ }^{5,6}$ Although early studies focused on long- and short-loop integrin-recycling pathways ${ }^{6}$, recent data have identified trafficking of the $\alpha 5 \beta 1$ integrin to and/or from late endosomal/lysosomal (LE/Lys) compartments as a key step in cell migration 7,8 . In addition, we found that the ECM molecule fibronectin (FN) is endocytosed and resecreted from a LE/Lys compartment to promote in vitro migration of fibrosarcoma, breast cancer $^{9}$ and epicardial ${ }^{10}$ cells. Finally, although the cargoes are unknown, additional studies pinpoint secretion from LE/Lys compartments as important for leukocyte chemotaxis and epithelial migration ${ }^{11,12}$. Thus, current data suggest that a key decision step in cell migration may be whether otherwise degradatory LE/Lys compartments fuse with the plasma membrane to release important cargo, such as ECM components and their receptors.

Among the components of LE/Lys, compartments that might affect cellular migration are exosomes. Exosomes are small secreted vesicles that carry bioactive cargoes, including growth factors, angiogenic factors, transmembrane receptors, proteinases, ECM molecules and RNAs ${ }^{13}$. It has been shown that purified exosomes can promote adhesion and motility of cells ${ }^{14,15}$; however, it is unclear how essential the process of exosome secretion is to cell migration or how it might affect underlying processes such as polarization. It is also unclear how exosome and/or LE/Lys secretion might impact cell migration through complex tissue environments.

To understand how LE/Lys secretion and exosomes control in vivo cell motility, we performed xenograft tumour cell motility studies in the chorioallantoic membrane (CAM) of chick embryos. This system is highly advantageous because it enables high-resolution live-imaging studies of cell migration through a physiologic collagen-rich stromal tissue environment ${ }^{16-18}$. We find that exosome secretion is critical for persistent directional migration of tumour cells in the chick CAM, likely due to stabilization of leading-edge protrusions. We further identify exosomes as critical carriers of ECM that promote adhesion assembly, a key step in leading-edge stabilization ${ }^{19-21}$. Targeting of the ECM molecule FN to exosomes depends on specific binding to integrin receptors, ensuring that ECM secreted on exosomes will match cellular receptors.

\section{Results}

Endolysosomal secretion controls HT1080 migration in vivo. To determine the impact of LE/Lys secretion on in vivo motility, we inhibited two canonical regulators of LE/Lys secretion, Rab27a and Synaptotagmin-7 (Syt7), in HT1080 fibrosarcoma cells. Rab27a controls plasma membrane docking of multivesicular late endosomes (MVE) ${ }^{22}$ whereas Syt7 controls fusion of LE/Lys compartments, including MVE, with the plasma membrane ${ }^{23,24}$. Syt7 and Rab27a expression were stably downregulated by the expression of specific targeting short hairpin RNAs (shRNAs; Fig. 1a). Green fluorescent protein (GFP)-expressing HT1080 cells carrying the scrambled control (HT1080 $0^{\text {Scrambled) }}$ or targetspecific shRNA (HT1080 Rab27aKD and HT1080 $0^{\text {Syt7KD }}$ ), were imaged intravitally after local engraftment or intravenous (i.v.) injection into the CAM of chick embryos (Fig. 1b) ${ }^{17,18}$. Primary tumours formed after engraftment enable the visualization of cells migrating away from the tumour periphery, while i.v. injection enables high-resolution single-cell tracking of individually extravasated cells. After engraftment, the HT1080 Scrambled, HT1080 Rab27aKD or HT1080 ${ }^{\text {Syt7KD }}$ cells all formed tumours in the CAM with little obvious difference in tumour size. Examination of the invasive front of each tumour at 4 days after inoculation revealed that fewer KD cells had migrated away from the tumour as compared with control cells (Fig. 1c,d). Following i.v. injection, cells that have extravasated from the bloodstream into the CAM grow to form colonies. Consistent with the previous studies ${ }^{18}$, individual control HT1080 cells had an elongated morphology and migrated rapidly through the CAM to form dispersed, loosely connected colonies (Fig. 1e,f). In contrast, extravasated HT1080 Rab27aKD or HT1080 $0^{\text {Yyt7KD }}$ cells exhibited a rounded morphology and formed few colonies that were overall larger in size, suggesting ongoing proliferation but defective migration (Fig. 1e,f) 18,25 .

Endolysosomal secretion promotes persistent and fast motility. To directly measure cancer cell motility in vivo, we performed time-lapse imaging of cancer cells migrating within the

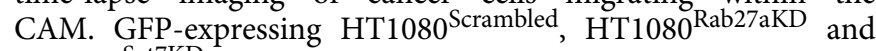
HT1080 $0^{\text {Syt7KD }}$ were imaged in the CAM for $16 \mathrm{~h}$ during a period of 1-3 days after i.v. injection. Analysis of the movies revealed that $\mathrm{HT} 1080^{\text {Scrambled }}$ exhibited polarized migration, allowing them to move in a directionally persistent manner. Conversely, HT1080 Rab27aKD and HT1080 Syt7KD cells that initially moved in one direction would frequently stop and reverse course (representative cell tracks shown in Fig. 2a, see also Supplementary Movies 1 and 2), leading to diminished overall displacement from the initial position (Fig. 2b,c). HT1080 Rab27aKD and HT1080 $0^{\text {Syt } 7 K D}$ cells also exhibited an overall defect in the total path length travelled by the cells, regardless of the directionality (Fig. 2b,c). To determine whether there was a distinct defect in directionality in $\mathrm{KD}$ cells, separate from any defects in overall speed of movement, the persistence index was calculated by dividing the displacement rate by cell speed (Fig. 2d,e). Fully persistent movement would result in a straight migration track and give a persistence index of 1 . Control cells had a persistence index of $\sim 0.4$. By contrast, HT1080 Rab27aKD and HT1080 $0^{\text {Syt } 7 \mathrm{KD}}$ cells had persistence indexes of $\sim 0.2$, indicating a strong defect in directionally persistent migration, independent of defects in cell speed (Fig. 2e). Wind-Rose plots of representative cell tracks further illustrate that endolysosomal secretion promotes persistent and efficient in vivo migration (Fig. 2f). A similar phenotype was observed in HEp3 squamous carcinoma cells (Supplementary Fig. 1).

Endolysosomal secretion controls protrusion dynamics. To further identify cellular features of migration regulated by endolysosomal secretion, we performed high-magnification livecell imaging of cells migrating in the CAM. Analysis of the cellular morphology revealed that $\mathrm{HT} 1080^{\text {Scrambled }}$ cells were much more elongated than cells inhibited for LE/Lys secretion (Fig. 3a,b, see also Supplementary Movie 3). It was also apparent that HT1080 Rab27aKD and HT1080 $0^{\text {Syt } 7 \mathrm{KD}}$ cells made more numerous but shorter-lived protrusions. Quantitation of the protrusion dynamics revealed that KD cells had a $50 \%$ increase in 
the number of protrusions formed per frame during migration (Fig. 3c), along with a decrease in the length and persistence of protrusions (Fig. 3d,e). These data indicate that LE/Lys secretion strongly contributes to establishment of a persistent leading-edge protrusion in vivo, which is critical for maintaining the cell directionality during migration. Interestingly, these results are reminiscent of our previous in vitro finding that endolysosomal recycling of ECM promotes the stabilization of leading-edge a

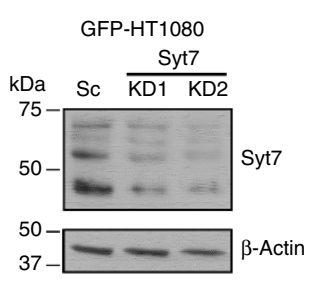

b

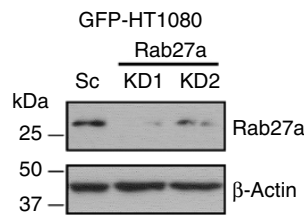

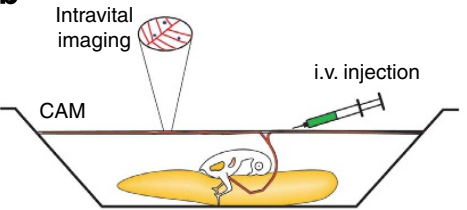

C

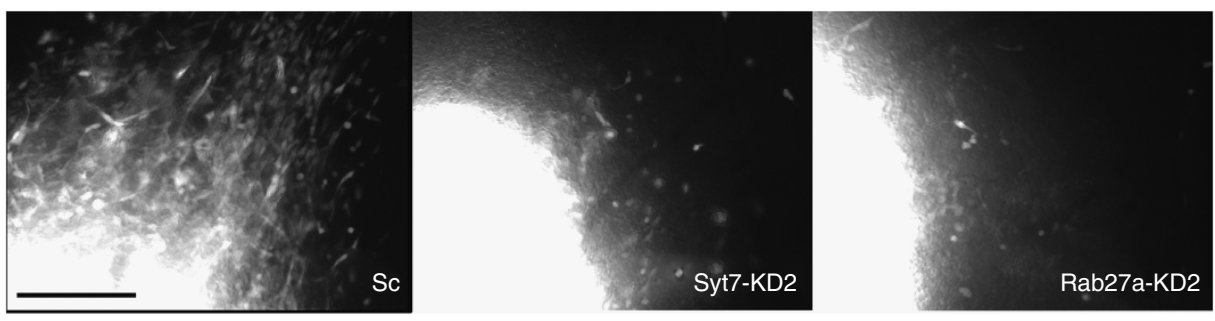

d
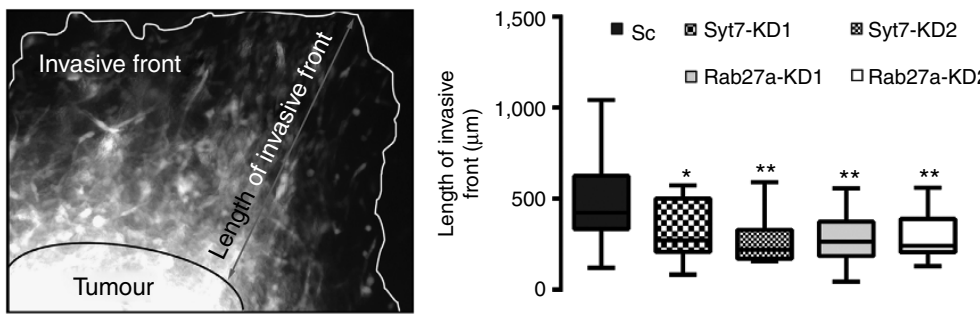

e
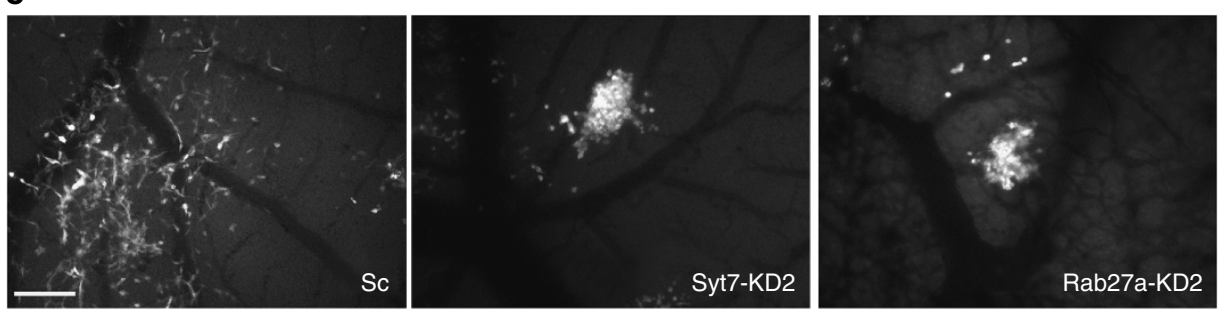

$\mathbf{f}$
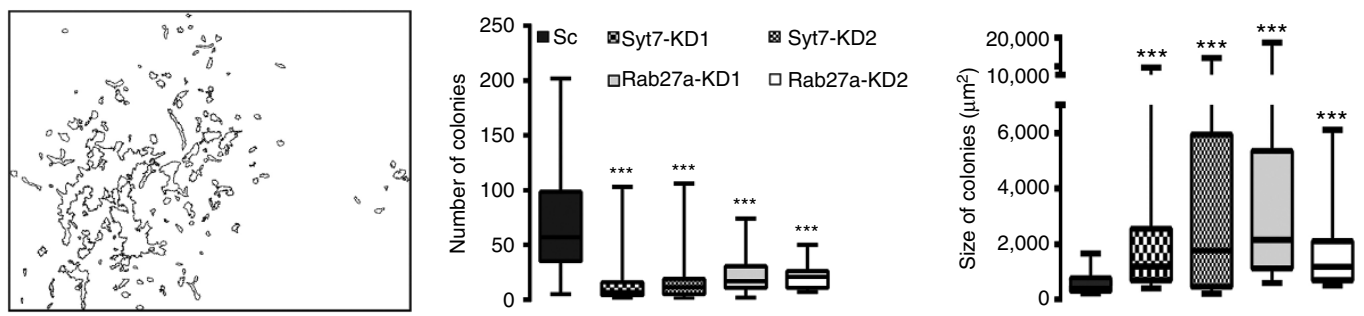

Figure 1 | Endolysosomal secretion controls cancer cell motility in vivo (a) Western blots for the expression of synaptotagmin-7 (Syt7) and Rab27a in GFP-expressing HT1080 cells. Sc, scrambled control; KD, knockdown. (b) A schematic cartoon of the chick CAM model. GFP-expressing HT1080 human fibrosarcoma are injected either directly into the CAM or i.v. (c) Primary tumours formed on the CAM through subcutaneous injection. Note the lack of cell migration away from Syt7- and Rab27a-KD tumours. Representative images from $\geq 13$ tumours for each condition. (d) Quantitation of the length of the migration front, defined as longest distance away from the primary tumour, as shown in image on left. (e) Migration away from colonies formed by extravasated cancer cells on the CAM through i.v. injection. Representative images from $\geq 26$ fields. (f) Quantitation of colony number and size/image from thresholded images similar to shown example. Each image for KD is representative of two different targeting shRNA-cell lines (KD1, KD2) and all data are from $\geq 3$ independent experiments. Box and whiskers plots are used where the box indicates the 25-75th percentile and whiskers indicate the 5-95th percentile and the black line indicates the median. ${ }^{\star} P<0.05 ;{ }^{\star \star} P<0.01$; ${ }^{\star \star \star} P<0.001$ compared with Sc using Mann-Whitney $U$-test. Scale bars, $200 \mu \mathrm{m}$. 
a
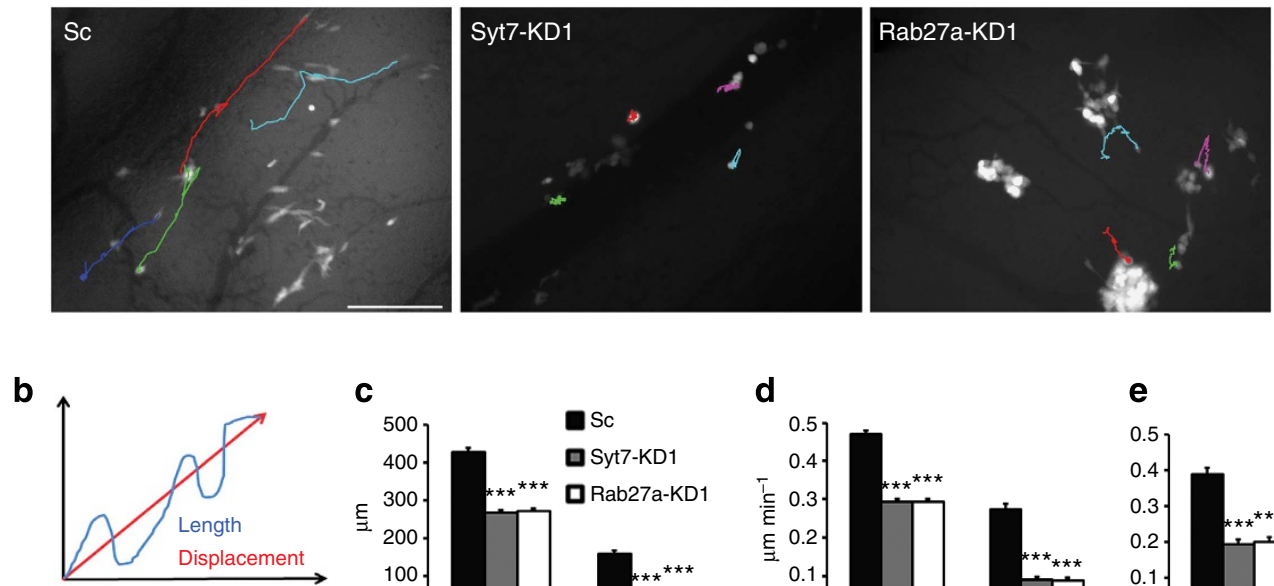

C

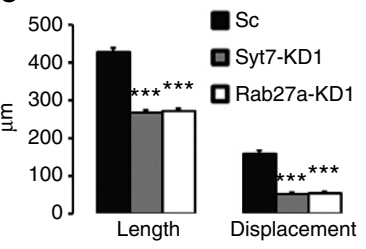

d

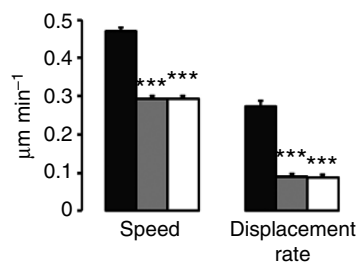

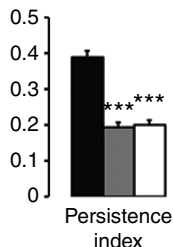

f

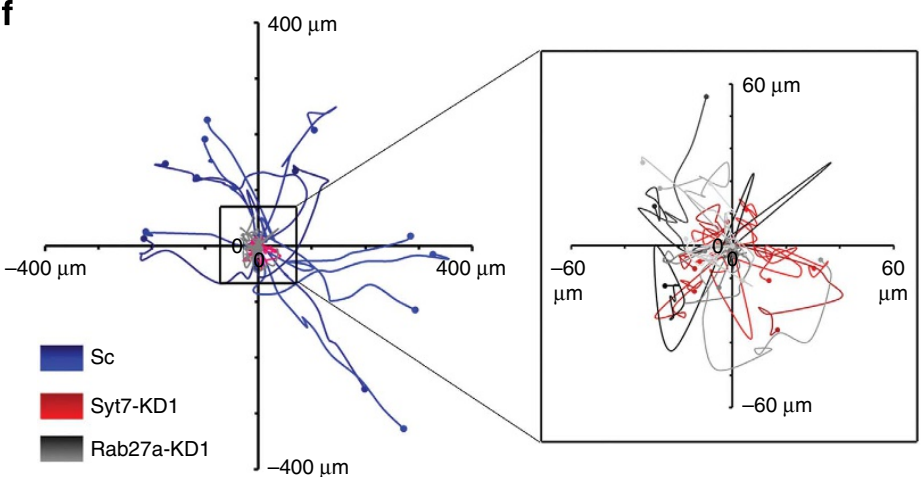

Figure 2 | Endolysosomal secretion is critical for persistent and fast migration (a) Representative images of HT1080 cells migrating within the CAM. Representative cell tracks are shown. Scale bar, $200 \mu \mathrm{m}$. See also Supplementary Movies 1 and 2. (b) Depiction of total path length (Length, blue curved line) and productive migration (Displacement, red arrow) calculations from cell tracks. (c) Length and displacement plots. (d) Speed, calculated by length divided by time. Displacement rate, calculated by displacement divided by time. (e) Persistence index, calculated as displacement rate divided by speed. (f) Wind-Rose plots from cell tracks. Magnification of boxed area shown on right. Error bars, s.e.m. from four independent experiments $\left(n>130\right.$ cells for each cell line). ${ }^{\star \star \star} P<0.001$ compared with Sc by Student's $t$-test.

protrusions $^{9}$. To determine if Rab27a and Syt7 also control the stability of protrusions in vitro, we performed live imaging and kymography analysis of the movies. We found a similar in vitro phenotype, with HT1080 Rab27aKD and HT1080 making an increased number of short-lived protrusions (Fig. 3f,g). The strong similarity between the in vitro and in vivo phenotypes suggests that we can exploit powerful available in vitro techniques to identify the mechanisms by which endolysosomal secretion controls cell motility.

Exosomes promote effective cell motility by carrying ECM. A specialized endolysosomal compartment that could potentially control cell migration is the MVE, which on fusion with the plasma membrane releases exosome vesicles to the extracellular milieu $^{13}$. To confirm that exosome secretion is inhibited in HT1080 Rab27aKD and HT1080 Syt7KD cells, exosomes were purified from conditioned media by serial centrifugation and analysed by NanoSight particle analysis and immunoblotting for the exosome marker flotillin and the ECM molecule FN. NanoSight analysis revealed that HT1080 Rab27aKD and HT1080 ${ }^{\text {Syt7KD }}$ cells secreted 2.2- to 3-fold fewer exosomes/cell than control cells (Fig. 4a). Consistent with the reduction in exosome secretion, the immunodetection of flotillin and FN was similarly reduced in exosome preparations from HT1080 Rab27aKD and HT1080 ${ }^{\text {Syt7KD }}$ cells (Fig. 4b, top panel). However, when an equal number of vesicles was loaded on blots, there was no difference in FN or flotillin levels (Fig. 4b, bottom panel).

To determine if autocrine secretion of exosomes was required for in vitro cell motility, live imaging of $\mathrm{HT} 1080^{\text {Scrambled }}$ and HT1080 Rab27aKD cells was performed in the absence or presence of purified exosomes. In the absence of exogenously provided exosomes, HT1080 Rab27aKD cells had a defect in the speed of migration (Fig. 4c,d, 'Exo 0' condition). However, unlike the migration in vivo, HT1080 Rab27aKD did not exhibit diminished persistence compared with $\mathrm{HT} 1080^{\text {Scrambled }}$, as quantitated by the persistence index (compare Fig. 4e, 'Exo 0', to in vivo migration results in Fig. 2e) and visualized in Wind-Rose plots (Fig. 4f). We speculate that this difference reflects the presence of directional cues in vivo that are not present in our in vitro motility environment.

Exosomes have the potential to control multiple aspects of cell motility, including stimulation of extracellular receptor signalling and as carriers of ECM to promote adhesion formation $14,15,26,27$. As a first test of these possible roles, exosomes purified from control HT1080 cells were added to the cells in two formatseither added into the media at the time of migration like a growth factor (Fig. 4c) or coated overnight onto tissue culture plates similar to ECM coatings (Fig. 4d). On addition of purified exosomes, HT1080 ${ }^{\text {Scrambled }}$ cells exhibited increased migration 
a
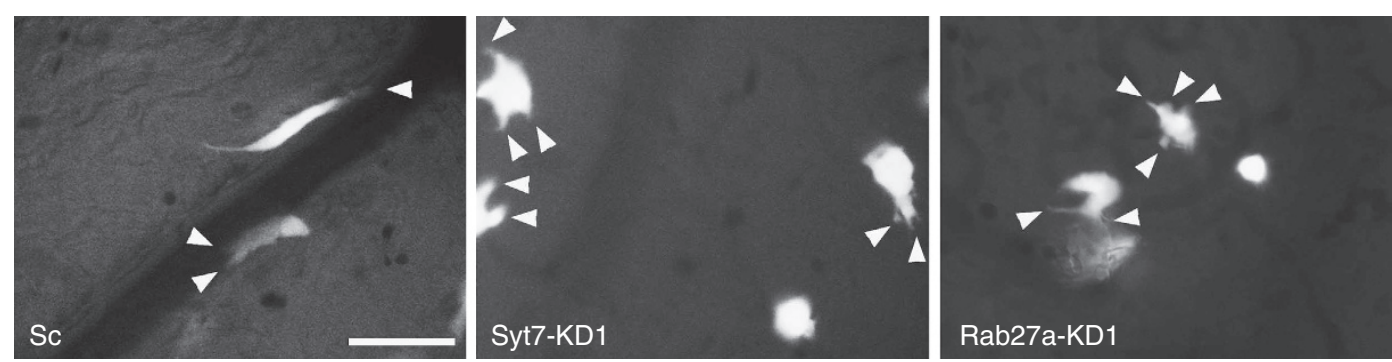

b

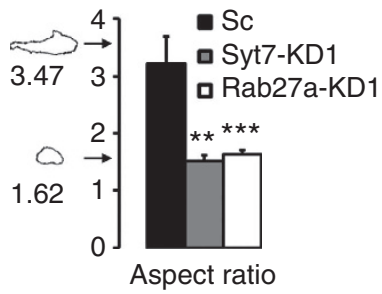

f
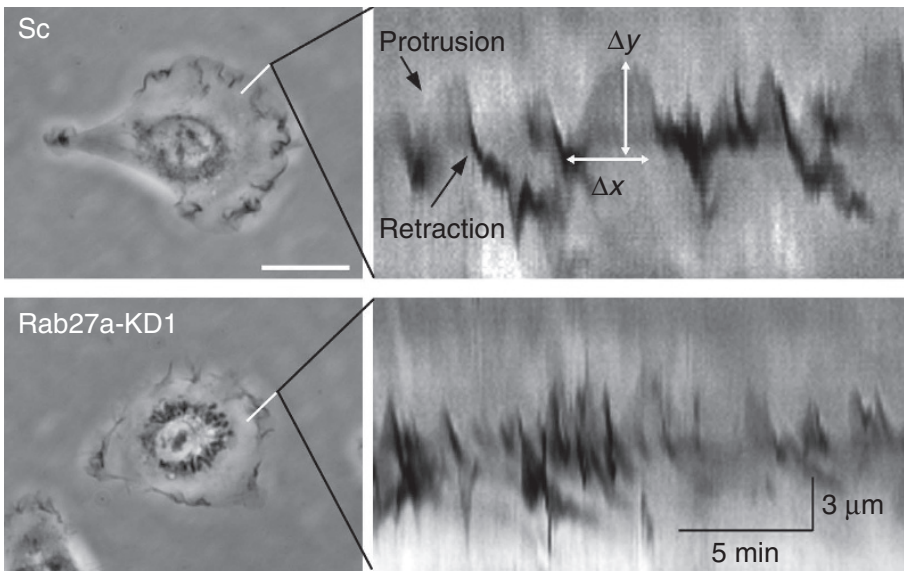

C

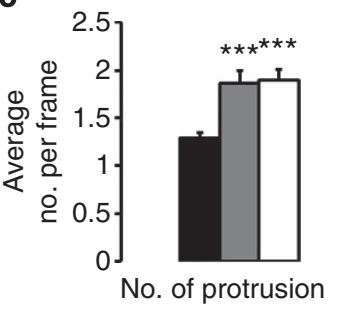

d

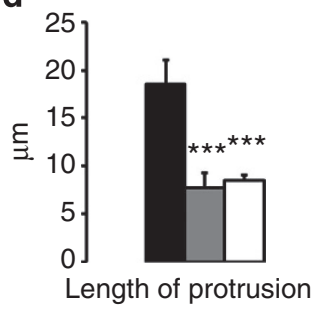

e

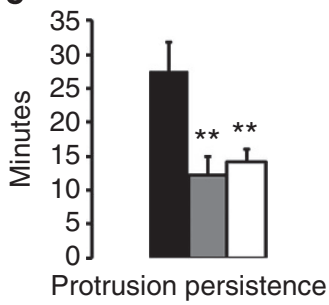

g
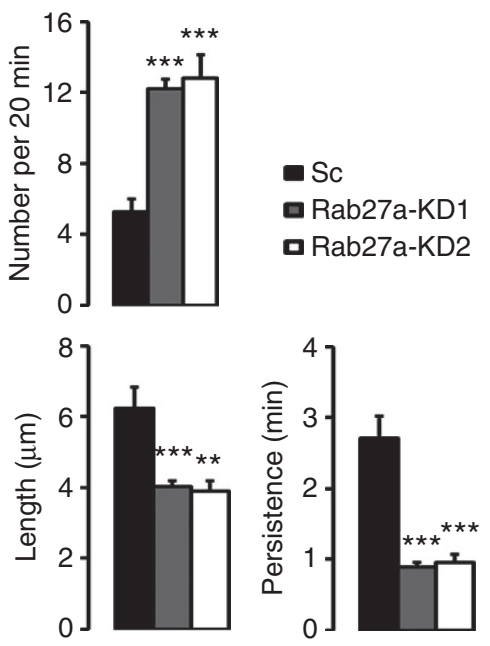

Figure 3 | Endolysosomal secretion controls protrusion dynamics in vivo and in vitro (a-e) In vivo protrusion dynamics of HT1080 cells in the CAM (a) Representative images. Arrowheads indicate protrusions. Movies were recorded every 2 min for 60 min. Scale bar, $50 \mu \mathrm{m}$. See also Supplementary Movie 3. (b) Aspect ratio was calculated as length of major axis divided by length of minor axis of cell. Representative cell morphologies for high (3.47) and low (1.65) aspect ratios are shown at the left of the bar graphs. (c) Number of protrusions per cell was counted at each time frame (2 min) and averaged over movies (60 $\mathrm{min})$. (d) Length of each protrusion. (e) Persistence of each protrusion was measured as the time between appearance and disappearance of each protrusion. $n>30$ cells for each cell line. (f,g) In vitro protrusion dynamics of HT1080 cells on tissue culture-treated dishes. (f) Representative kymographs. Note definitions of protrusion persistence (length of time that a protrusion lasts before retraction, $\Delta x$ ) and length of protrusion ( $\Delta y$ ). Scale bar on Sc cell image indicates $30 \mu \mathrm{m}$. Scale bars on kymograph indicate time and distance axes. (g) Quantitation of number of protrusions/time, length of protrusion and protrusion persistence. $n \geq 12$ cells for each condition. Error bars, s.e.m. from three independent experiments. ${ }^{\star} P<0.05 ;{ }^{\star \star} P<0.01$; ${ }^{\star \star \star} P<0.001$ compared with Sc by Student's $t$-test.

speed, especially when exosomes were coated onto culture dishes. Interestingly, the speed defect of HT1080 Rab27aKD cells was fully rescued on exosome-coated plates (Fig. $4 \mathrm{~d}$ ) but not with the addition of exosomes into media containing exosome-depleted serum (Fig. 4c). These data suggest that deposition of exosomes to the substrata where it is accessible to the basal cell surface is important for their ability to promote cell migration.

The presence of FN on exosomes (Fig. 4b), the lamellipodial stability defect of KD cells (Fig. 3) and the selective rescue of Rab27a-KD cells on exosome-coated plates (Fig. 4d) suggest that exosomes may serve a critical function in providing matrix attachment for migrating cells. By immunoprecipitation, FN is on the outside of exosomes, indicating that it is indeed of the expected topology to support adhesion and migration
(Supplementary Fig. 2). To determine whether the presence of FN on exosomes is important for their ability to support migration, we prepared FN-depleted exosomes by culturing control cells for 10 days in FN-depleted serum before collecting 48-h conditioned serum-free media for exosome purification. This procedure led to a 2.5 -fold decrease in the FN content of exosomes, compared with control preparations (Fig. 4g). When coated onto culture dishes and tested in single-cell motility experiments, FN-depleted exosomes were less efficient at rescuing single-cell motility than control exosomes (compare Fig. $4 \mathrm{~h}$ to Fig. 4d). Thus, while $10 \mu \mathrm{g} \mathrm{ml}^{-1}$ coating of control exosomes fully rescued motility of Rab27a-KD cells, twice as many FN-depleted exosomes were required to rescue their motility. The rescue of Rab27a-KD cell motility by $20 \mu \mathrm{gml}-1$ 
a

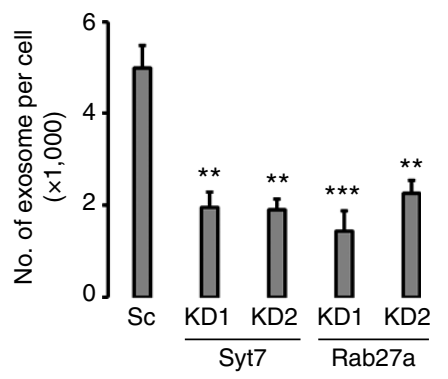

b

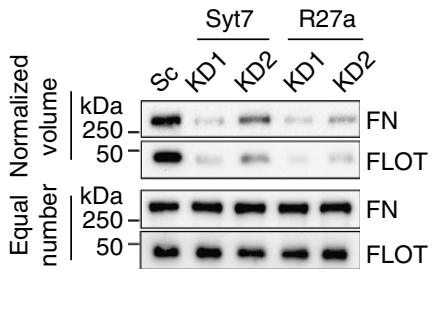

c

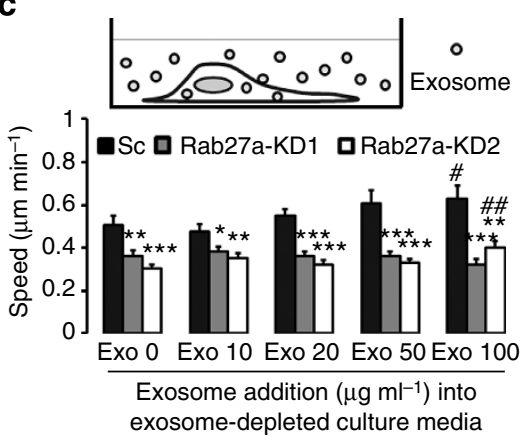

d

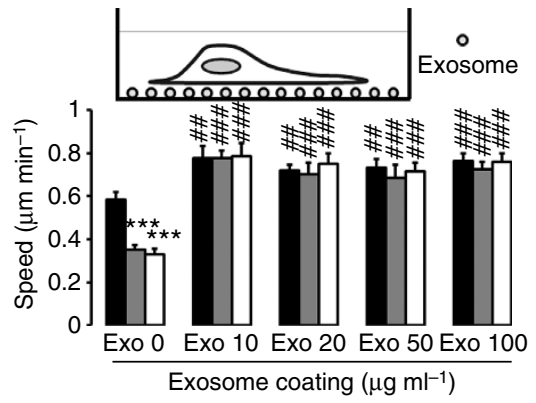

e

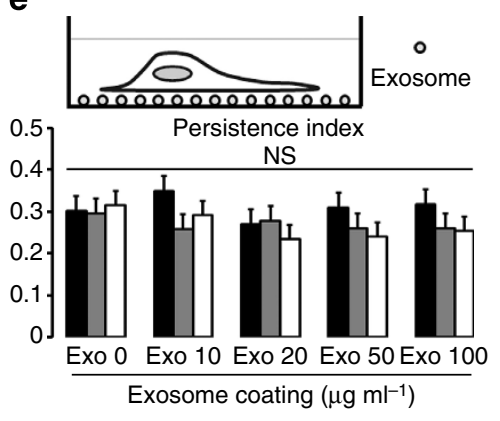

g

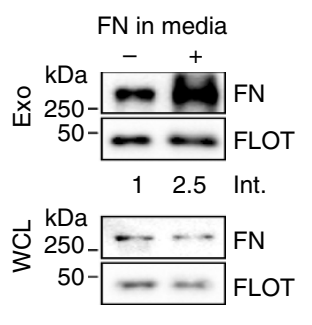

f

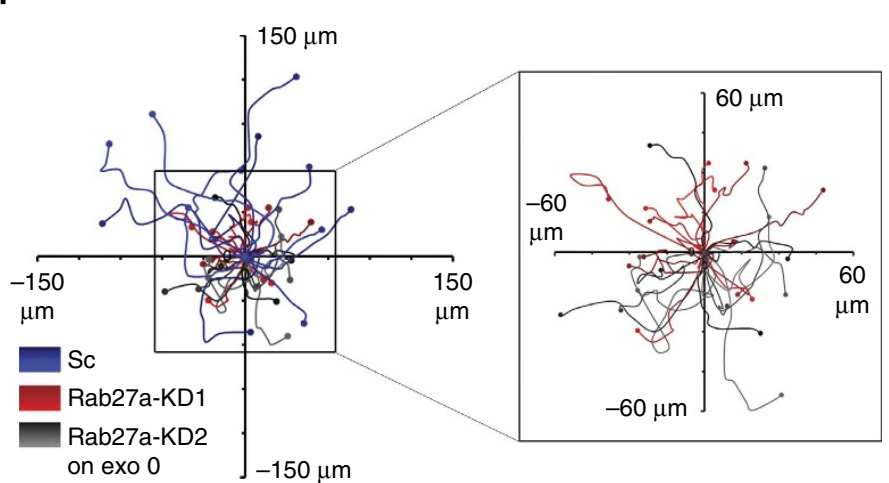

g
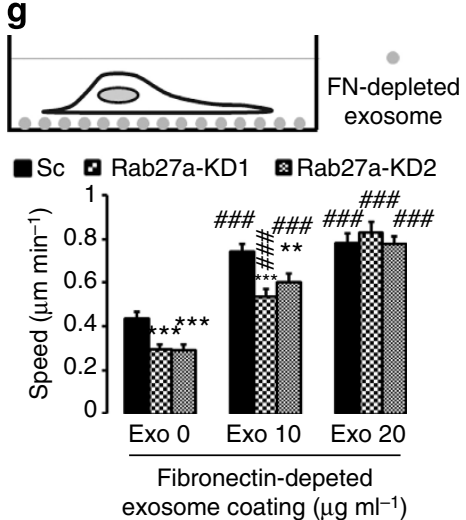

Figure 4 | Exosome secretion promotes cell migration. (a) Average number of exosomes secreted from endolysosomal secretion-inhibited HT1080 cell lines from $n \geq 3$ experiments. (b) Western blots of exosome cargos. FLOT, flotillin. (c) Migration speed of Rab27a-knockdown (KD) HT1080 cells in exosome-depleted media \pm add-back of purified exosomes (Exo), as indicated on tissue culture-treated plates. (d) Speed of Rab27a-KD cells on exosomecoated plates. (e) Persistence index calculated from experiments shown in d. (f) Wind-Rose plots for data shown in d, Exo 0. Magnification of boxed area is shown at the right. (g) Western blot for FN-depleted exosomes. Numbers below blot represent relative intensity (Int.) of FN (normalized to FLOT) from that blot. WCL, whole-cell lysates; (h) Single-cell migration assays on FN-depleted exosome-coated plates. Error bars, s.e.m., from $n \geq 29$ cells for each cell line from three independent experiments. ${ }^{\star} P<0.05$; ${ }^{\star \star} P<0.01$; ${ }^{\star \star \star} P<0.001$ compared with Sc by Student $t$-test. ${ }^{\#} P<0.05 ;{ }^{\# \# P<0.01 ;}{ }^{\# \# \# P<0.001}$ compared with same cell line on Exo 0 by Student's t-test. NS, not significant.

FN-depleted exosomes could be due to remaining $\mathrm{FN}$ on the FN-depleted exosomes or to other exosome cargoes.

The concomitant decrease in FN and the membrane marker flotillin in exosome preparations from Rab27a- and Syt7-KD cells that were loaded according to equal cell number (Fig. $4 \mathrm{~b}$, top blot) suggests that $\mathrm{FN}$ is not merely a contaminant of our ultracentrifugation (UC) preparation technique. Furthermore, the size distribution of exosomes and microvesicles in our UC fractions were as expected (Fig. 5a) and approximately threefold fewer MVs are secreted by our cells, suggesting that it is exosomes rather than microvesicles that promote cell motility in our system. However, to test the specificity of exosomes in carrying FN and promoting cell motility, we further purified our UC exosome preparations by sedimentation into a density gradient of Optiprep (DG exosomes). Western blot analysis of the fractions revealed that FN was purified in two peaks-one that included the exosome markers HSP70 and flotillin along with the FN-binding integrin $\alpha 5$ subunit and another peak that did not have any of those markers and probably represents FN fibrils not associated with exosomes (Fig. 5b). Microvesicle preparations contained no FN or HSP70 but did contain a small amount of flotillin and $\alpha 5$ integrin, which are both plasma membrane markers and may be found in both types of vesicles. To determine whether our Optiprep-purified exosomes could rescue cell motility defects of Rab27a-KD cells, we coated plates and performed the same live-imaging assay as we had performed with UC-exosomes. Compared with one-step UC-exosomes from the same conditioned media preparation, Optiprep-double purified exosomes were equipotent in their ability to rescue cell motility (Fig. 5d). By comparison, the microvesicle fraction from the same 
a
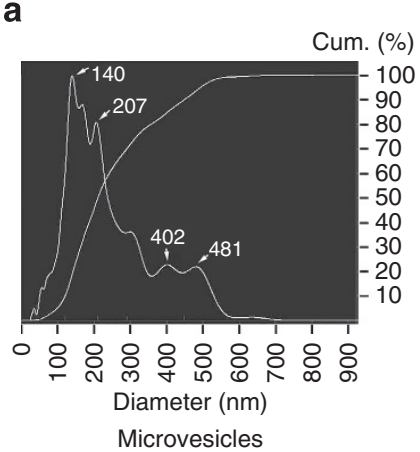

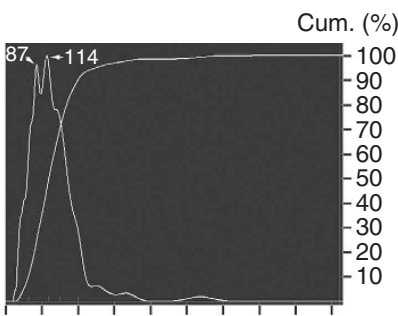

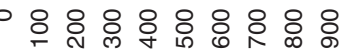
Diameter (nm)

Ultracentrifuged exosomes

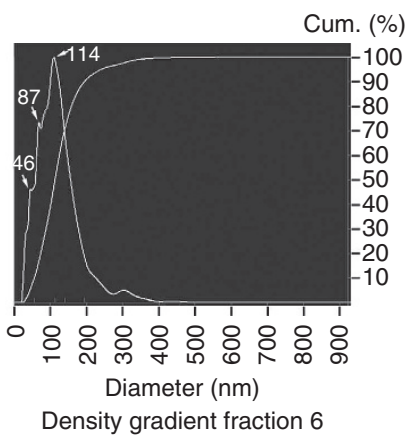

b

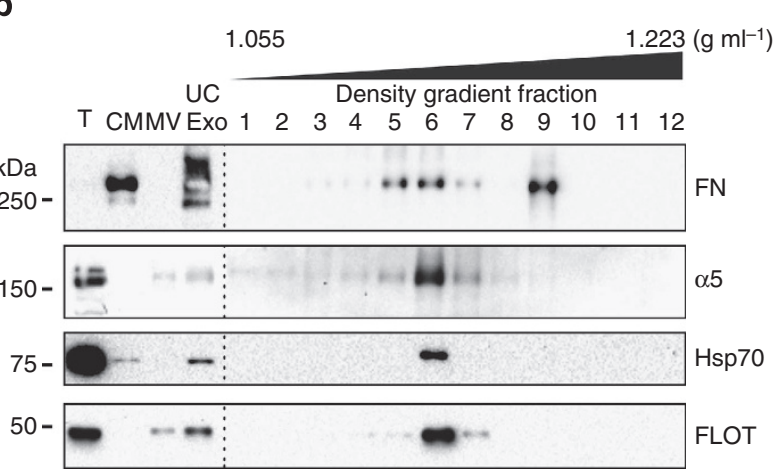

C

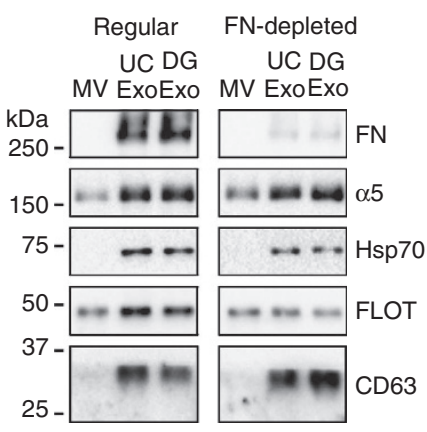

d
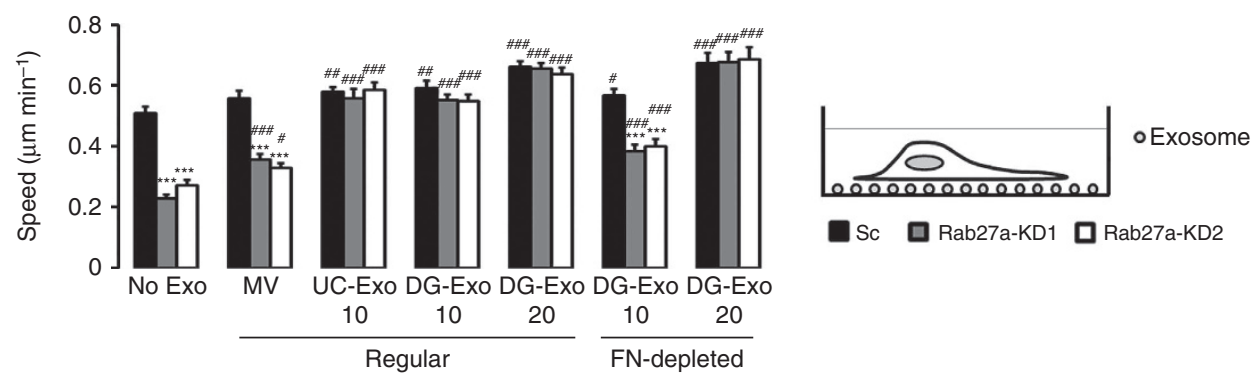

Exosome coating $\left(\mu \mathrm{g} \mathrm{ml}^{-1}\right)$

Figure 5 | Density gradient-purified exosomes have bound FN and rescue Rab27a-KD cell motility defects Extracellular vesicles were purified by ultracentrifugation. Microvesicle (Microvesicles) and exosome (Ultracentrifuged exosomes) fractions were analysed by NanoSight (a). Part of the Ultracentrifuged exosomes fraction was further purified by Optiprep density gradient centrifugation. (b) Western blot analysis of the fractions (Density gradient fractions 1-12) for exosome markers HSP70 and flotillin identified Fraction 6 as the major peak containing exosomes. NanoSight analysis of this fraction ((a): Density gradient fraction 6 ) showed a typical exosome size profile similar to the Ultracentrifuged exosomes profile, whereas Microvesicles fraction contained larger vesicles. Both FN and its receptor $\alpha 5$ integrin were present in the Fraction 6 exosome peak. FN was also present separate from exosome markers in Fraction 9. Conditioned media (CM) contained FN but the microvesicle (MV) fraction did not. T= Total cell lysate. Representative blots from $n=2$. (c) Western blot analysis of MVs, ultracentrifuge-purified exosomes (UC-Exo) and exosomes present in Fraction 6 density gradients (DG-Exo) from control cells cultured in normal media (Regular) or in FN-depleted media for 10 days. Equal numbers of vesicles were loaded in the gel lanes. Note that FN, HSP70 and CD63 were uniquely present in the exosome samples. Although the lanes are demarcated from each other due to intervening lanes not being shown, the regular and FN-depleted Exo lanes came from the same blot and are directly comparable for all antibodies. (d) The ability of various extracellular vesicles to rescue motility of Rab27a-KD cells was tested by coating $10 \mu \mathrm{g} \mathrm{ml} \mathrm{l}^{-1} \mathrm{UC}$-Exo, microvesicles purified from an equivalent number of cells that produced $10 \mu \mathrm{g} \mathrm{ml} \mathrm{I}^{-1} \mathrm{UC}$-Exo or 10 or $20 \mu \mathrm{g} \mathrm{ml} \mathrm{l}^{-1} \mathrm{DG}$-Exo. Similar to results with UC-Exo (Fig. 4d,h), twice as many DG-Exo purified from FN-depleted cells were required to rescue Rab27a-KD speed as Regular DG-Exo. Data from $\geq 30$ cells from $\geq 3$ independent experiments for each condition. Error bars indicate s.e.m. ${ }^{\star \star \star} P<0.001$ compared with $S c$ by Student's $t$-test. ${ }^{\#} P<0.05$; $\# \# P<0.01$; \#\#\# $P<0.001$ compared with the same cell line on Exo 0 by Student's $t$-test.

conditioned media was unable to rescue motility. As with the UC-exosomes (Fig. 4h), FN-depleted DG exosomes were less efficient in rescuing cell motility compared with control DG exosomes (Fig. $5 c, d$ ).

These data are consistent with our recent finding that recycling of endocytosed FN from a LE/Lys compartment drives cell motility ${ }^{9}$ and suggest that exosomes are key delivery vehicles for that FN. Consistent with this model, we tested whether endocytosed fluorescent FN was likewise secreted by HT1080 cells migrating in the chick CAM. Indeed, $24 \mathrm{~h}$ after i.v. injection of DyLight550-FN-loaded HT1080 cells into the chick CAM, we observed incorporation of the endocytosed FN into streak-like structures reminiscent of adhesions associated with cellular protrusions (Supplementary Fig. 3 and Supplementary Movie 4). 
To further verify that the secretion of exosomes rather than non-exosomal LE/Lys cargo is required for motility, we knocked down a component of the endosomal sorting complexes required for transport (ESCRT) machinery that is necessary for intraluminal vesicle formation within $\mathrm{MVE}^{28}$. Hepatocyte growth factor-regulated tyrosine kinase substrate (Hrs) is part of the ESCRT-0 complex, and inhibition of Hrs prevents exosome biogenesis (Supplementary Fig. 4, refs 23,29). As expected, Hrs $\mathrm{KD}$ diminished the release of exosomes into conditioned media (Supplementary Fig. $4 \mathrm{a}-\mathrm{c}$ ) and HT1080 $\mathrm{HrsKD}$ cells had motility defects in vivo (Supplementary Fig. 4d,e) and in vitro (Supplementary Fig. 4f, Exo 0 and $5 \mathrm{~g}$, FN 0 conditions). Consistent with our hypothesis that matrix-containing exosomes promote cell motility, the in vitro motility speed defect of HT1080 HrsKD cells was fully rescued on culture dishes coated with either exosomes (Supplementary Fig. 4f) or $10 \mu \mathrm{g} \mathrm{ml}^{-1}$ purified FN (Supplementary Fig. $4 \mathrm{~g}$ ).

Secreted exosomes promote assembly of nascent adhesions. Our data suggest that a major role of exosomes is to transport ECM and presumably regulate adhesions. To investigate whether exosome-containing MVE are transported to and secreted at specific cellular locations, we performed live confocal imaging of cells expressing the MVE marker mCherry-CD63. To simultaneously visualize adhesions, we expressed GFP-paxillin. As previously reported, CD63-positive tubules and vesicles were observed moving dynamically in cells (Fig. 6a and Supplementary Movie 5). In some cases, these vesicular structures appeared to target peripheral adhesions (Fig. 6a and Supplementary Movie 5, arrows); however, the majority of the CD63 was in endosomes away from the plasma membrane and some CD63-positive endosomes also carried paxillin (Fig. 6a and Supplementary Movie 5, arrowhead).

To definitively identify exosome secretion sites, we constructed a novel imaging probe, which has the $\mathrm{pH}$-sensitive GFP-pHLuorin moiety inserted into the first extracellular loop of CD63 (CD63pHLuorin). Because pHLuorin-GFP fluorescence is quenched at the acidic late endosomal $\mathrm{pH}$, but fluoresces brightly at the neutral $\mathrm{pH}$ of the extracellular environment ${ }^{30}$, it is ideal to track proteins that are exocytosed from endosomes. To validate this construct, we co-expressed it with mCherry-Rab5-Q79L, which both marks and leads to the enlargement of early-to-late-transitioning endosomes $^{31}$, in control and Rab27a-KD cells. Consistent with the construct marking secretory MVE, pHLuorin-CD63 fluorescence was present on the cell surface of control cells, but not of Rab27a-KD cells (Supplementary Fig. 5). In addition, Bafilomycin treatment of Rab27a-KD cells to inhibit acidification of endosomes reveals its presence in internal vesicles. Finally, since many Rab5-positive endosomes will not be fully acidified yet, note the internal co-localization of pHLuorin-CD63 with some Rab5Q79L endosomes in control cells but less co-localization in Rab27a-KD cells, which should have an accumulation of secretory MVE downstream of early endosomes due to the docking defect ${ }^{22}$.

To visualize exosome secretion in migrating cells, wide-field epifluorescence movies of HT1080 cells expressing pHLuorinCD63 and mCherry-paxillin migrating in two-dimensinal were performed (Fig. 6b and Supplementary Movie 6). Overall, pHLuorin-CD63 fluorescence was observed at the front of the cell in thick ruffles and at the back of the cell at attachment sites. At the back of the cell, a striking feature was prominent 'slime trails' of fluorescence that were frequently left behind the migrating cells (Fig. 6b,c). Examination of phase-contrast images from the same movies reveals that the rear of the cell is far in advance of the 'slime trails' and does not reveal any apparent bits of torn-off plasma membrane (Fig. 6b). Furthermore, examination of mCherry-paxillin fluorescence gathered at the same time shows that only green fluorescence is present in the trail (Fig. $6 \mathrm{~b}$ and Supplementary Movie 6). These data suggest that the primary source of the slime trail is exosomes and not torn-off plasma membrane or large adhesions left behind. These trails appeared to serve an adhesive function based on attachment to membrane at the rear of the cell that is particularly evident in the movies (Supplementary Movie 6). To further analyse the localization of pHLuorin-CD63 with respect to leading-edge protrusions, higher speed movies were taken. Kymographs showing protrusion at the leading edge over time were obtained and revealed that pHLuorin-CD63 was not enriched in the initial flat lamellipodial protrusions (Fig. 6c and Supplementary Movie 7). Instead there was an increased fluorescence in membranes that ruffled back, which could be due to membrane thickness or adhesion of exosomes after the initial membrane protrusion.

To further define the relationship between exosome secretion and adhesion formation, we performed live total internal reflected fluorescence (TIRF) microscopy of cells expressing both pHLuorin-CD63 and mCherry-paxillin (Fig. 7a and Supplementary Movie 8). To visualize newly forming adhesions, movies were taken in actively spreading cells. Interestingly, bursts of pHLuorin-CD63 closely preceded adhesion formation. Analysis of these data revealed that $93 \%$ of adhesions were preceded by a burst of CD63-pHLuorin, with a median of $2 \mathrm{~min}$ lag between CD63 appearance and adhesion formation (Fig. 7b,c).

To specifically test the role of exosome secretion in adhesion formation, we analysed the dynamics of adhesions in GFP-paxillin-expressing control and Rab27a-KD cells. Confocal movies taken in actively spreading cells revealed that Rab27a-KD cells have a selective defect in the rate of adhesion assembly (Fig. 7d,e and Supplementary Movie 9). There was no difference in the rate of adhesion disassembly (Fig. 7f) or in the overall number of peripheral adhesions per frame of the movies $\left(25.16 \pm 4.37\right.$ for HT1080 $0^{\text {Scrambled }}, 27.65 \pm 3.08 \quad(P=0.3229$ compared with scrambled) for HT1080 Rab27aKD1 and $30.40 \pm 4.24 \quad(P=0.2112$ compared with scrambled $)$ for HT1080 Rab27aKD2, $P$ by Student's $t$-test). Overall, the slow adhesion assembly time led to a significant prolongation of the overall adhesion lifetime (average lifetime $t_{1 / 2}$ for HT1080 Scrambled adhesions $=7.0 \pm 0.4 \mathrm{~min}$, compared with HT1080 ${ }^{\text {Rab27aKD1 }}=10.7 \pm 0.8 \mathrm{~min} \quad(P<0.002$, Student's $t$-test $)$ and HT1080 Rab27aKD2 $=10.4 \pm 1.0 \mathrm{~min} \quad(P<0.002$, Student's $t$-test). These data are consistent with the known relationship between dynamic adhesions and fast migration speeds ${ }^{32-34}$.

FN is targeted to exosomes by interactions with integrins. Our data indicate that FN becomes specifically targeted to exosomes in a form that promotes efficient adhesion formation. Soluble FN becomes converted to a fibrillar, adhesion-promoting form in association with cellular integrin receptors ${ }^{35}$, which are also frequent exosome cargos. To test whether targeting of FN to secreted exosomes depends on binding to integrins, we inhibited FN-integrin interactions and assessed the resulting FN content of exosomes. Treatment of HT1080 cells with integrin-binding RGD peptide for $48 \mathrm{~h}$ greatly decreased the FN content of exosomes compared with cells treated with control RGE peptide (Fig. 7g). Likewise, inhibition of the FN-binding integrin $\alpha 5 \beta 1$ by KD of $\alpha 5$ also decreased the FN content of exosomes (Fig. $7 \mathrm{~g}$ ). These data indicate that $\mathrm{FN}$ is targeted to exosomes by binding to cognate cellular integrin receptors, and then secreted in adhesive form to promote adhesion formation and effective cell motility.

\section{Discussion}

Cell movement through tissues is a complex process that requires a coherent response to potentially divergent tissue cues. Here we 
a

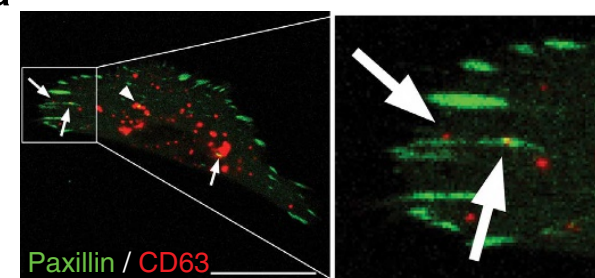

b
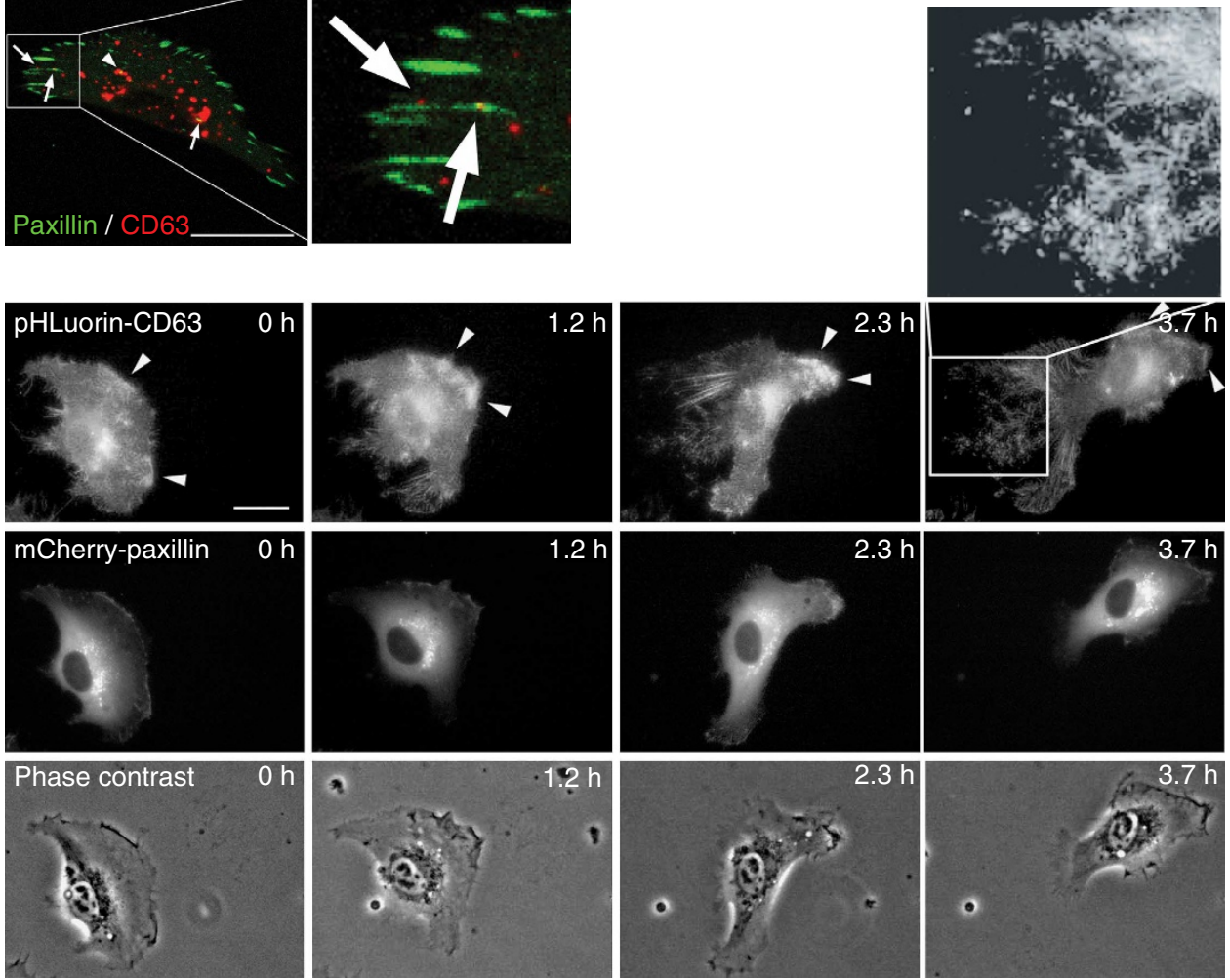

C
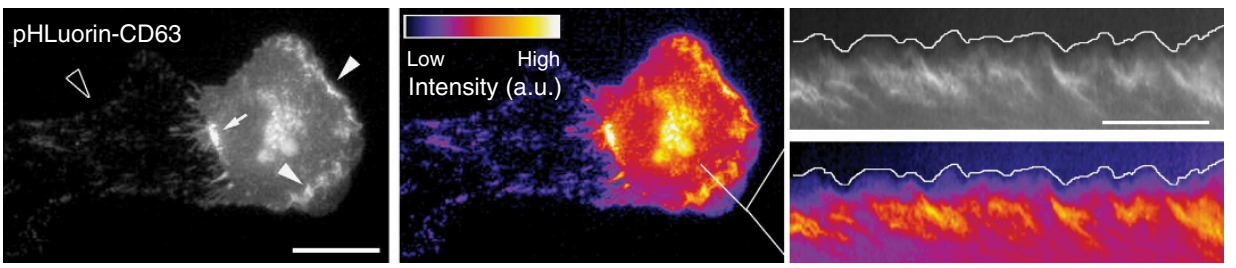

Figure 6 | Adhesive exosome trails are left behind migrating cells (a) Confocal image of mCherry-CD63 (red) and GFP-paxillin (green)-expressing HT1080 cell from a representative movie from $n=17$ movies on FN-coated $\left(1 \mu \mathrm{g} \mathrm{ml}{ }^{-1}\right.$ ) MatTek dishes. Arrows indicate co-localization of mCherry-CD63 with adhesions, whereas an arrowhead indicates localization of paxillin within endosomes. Magnification of boxed area is shown right. See also Supplementary Movie 5. (b) Image series from a representative epifluorescence movie (1 frame per 2 min, from $n=10$ movies) of a migrating HT1080 cell on FN-coated $\left(1 \mu \mathrm{g} \mathrm{ml}^{-1}\right)$ MatTek dishes transiently transfected with pHLuorin-CD63 to show sites of exosome secretion. Note CD63 fluorescence in ruffles at the front of the cell (arrowheads) and in an adhesive trail left behind the cell. Zoom is brightened and shows adhesive trail. See also Supplementary Movie 6. (c) Image from a representative epifluorescence movie with a rapid frame rate ( 1 frame per $6 \mathrm{~s}$, from $n=20$ movies) to capture the dynamics of pHLuorin-CD63 in protrusions. Filled arrowheads indicate fluorescence in ruffles, whereas open arrowhead points to adhesive trail. The arrow points to rear fluorescence near retracting fibres. Heat map-coloured intensity is shown right. Grayscale and coloured kymographs show protrusion over time across the perpendicular line drawn in heat map image. Note lack of enhanced fluorescence at flat protrusion membrane edge, which is outlined by drawn white lines. See also Supplementary Movie 7. Scale bars, $30 \mu \mathrm{m}$.

identify exosome secretion as a key autocrine mechanism to reinforce directional cues and support productive migration (see model in Fig. 8). In vivo, inhibition of exosome formation, MVE docking or MVE fusion led to similar defects in cell migration speed and persistence. In vitro, purified exosomes, but not shed microvesicles, were able to rescue speed defects of exosome-inhibited cells. Both in vitro and in vivo, secretion of exosomes stabilizes nascent protrusions, a phenotype associated with cellular adhesion ${ }^{19-21}$. In vitro live-imaging experiments further demonstrated that a key function of exosomes is to promote adhesion assembly. A critical exosome cargo that drives the adhesion and speed of migrating cells is ECM, as demonstrated by the reduction in speed supported by FN-depleted exosomes and full rescue of speed defects in exosome-inhibited cells by purified FN. Altogether, our results demonstrate that autocrine exosome secretion promotes both efficient and directional cell motility and indicates that a major function of ECM in exosomes is to promote adhesion formation and enhance cell speed.

While exosomes have been implicated in cell motility previously $14,15,26$, it has not been clear how essential to the process they are or what specific steps are regulated. Our data now demonstrate that autocrine secretion of exosomes controls both directional persistence and speed of cells migrating in vivo. To understand in depth the role of exosome-delivered ECM in cell motility, we carried out a variety of live-imaging experiments to both visualize the spatiotemporal relationship between exosome secretion and motility structure formation and demonstrate specific defects of exosome-inhibited cells in adhesion assembly and cell speed but not in protrusion 


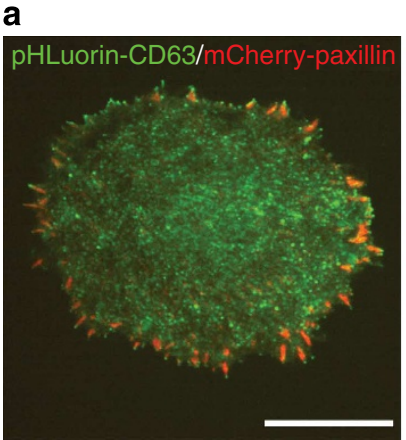

b

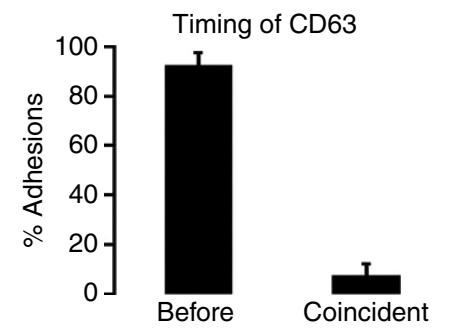

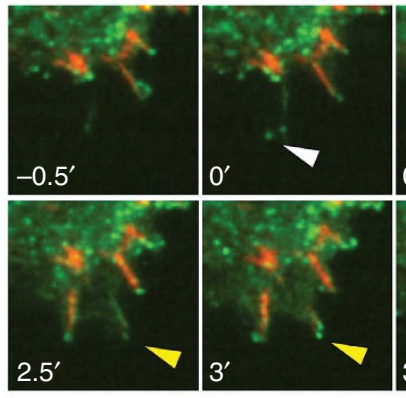
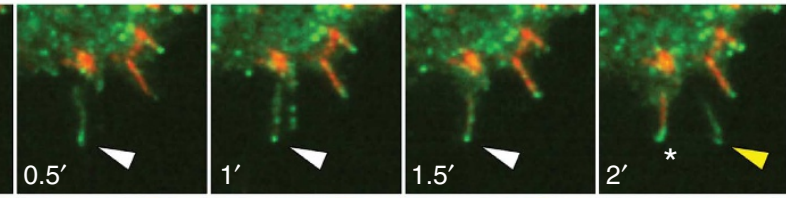

c

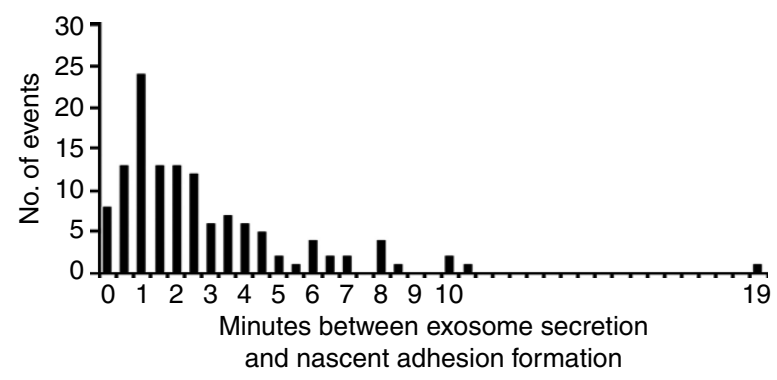

d
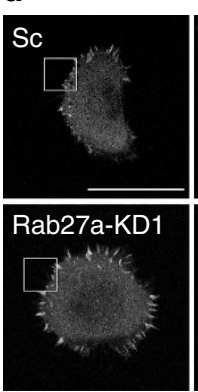

e
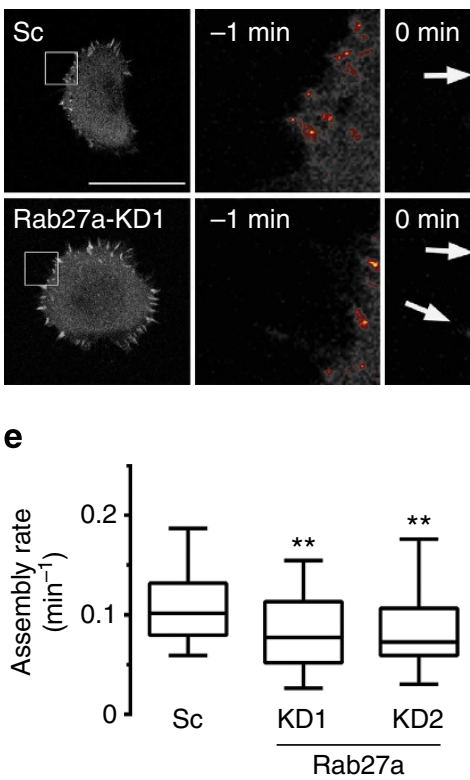

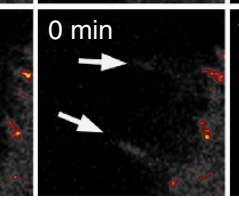

f

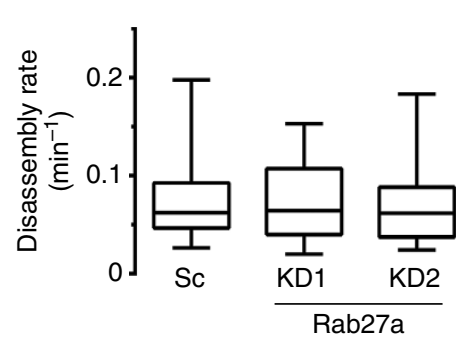

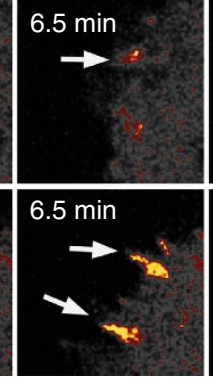
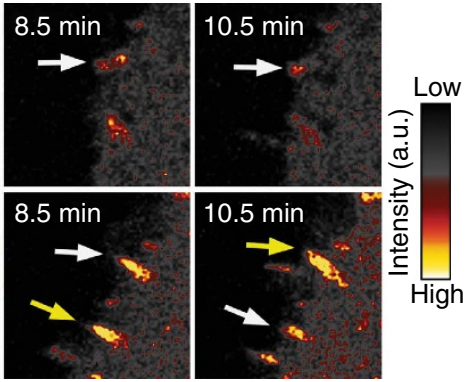

g

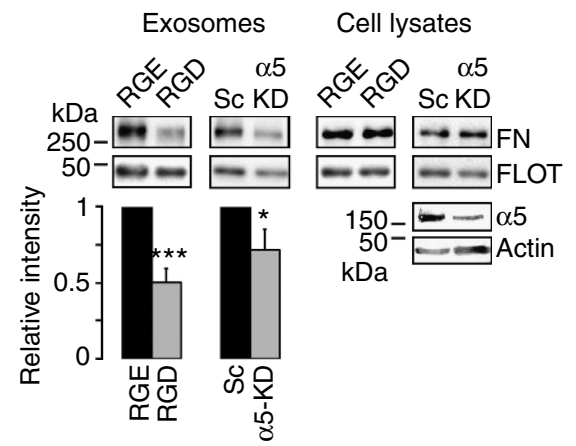

Figure 7 | Exosomes promote adhesion assembly (a) Image from TIRF movie of pHLuorin-CD63 (green) and mCherry-paxillin (red)-expressing HT1080 cell on FN-coated $\left(1 \mu \mathrm{g} \mathrm{ml}^{-1}\right)$ MatTek dishes. Time series shows bursts of pHLuorin-CD63 fluorescence (arrowheads) preceding adhesion formation (indicated by *, red paxillin fluorescence visible at that time). Two adhesion formation events are, respectively, indicated with white and yellow markers. Representative of $n=7$ movies. See also Supplementary Movie 8. (b) Quantitation of per cent of adhesions with a pHLuorin-CD63 fluorescence burst before or coincident with their formation. (c) Quantitation of the number of minutes between pHLuorin-CD63 appearance and adhesion formation.

(d) Representative time series of GFP-paxillin-marked adhesion assembly and disassembly for control (Sc) and Rab27a-KD HT1080 cells on FN-coated $\left(1 \mu \mathrm{gl}^{-1}\right)$ MatTek dishes. Magnifications of boxed area are shown at right by heat map coloration to show intensity. 0 min indicates start of adhesion formation. White arrows indicate example adhesions and yellow arrows indicate the peak adhesion intensity. Asterisk indicates a newly forming adhesion. Note that a longer period of time is required for adhesion assembly in KD cells. (e,f) Box-and-whisker plots of adhesion assembly (e) and disassembly (f) rates. Each box shows the 25-75th percentile and whiskers indicate the 5-95th percentile and the black line indicates the median. Analysis from $>30$ adhesions for $\mathbf{e}$ and $>40$ adhesions for $\mathbf{f}$ from three to eight cells per condition. See also Supplementary Movie 9. (g) Western blot analysis of FN association with exosomes. GRGESP (100 $\mu \mathrm{M}$; RGE, control) or GRGDSP (100 $\mu \mathrm{M}$; RGD, integrin-binding) peptides were added to cultured HT1080 control cells $(\mathrm{Sc})$ for $48 \mathrm{~h}$ before exosome isolation. Equal exosome numbers were loaded on western blots. Quantitations show FN intensity normalized to flotillin intensity as a loading control from $n \geq 4$ western blots. $\alpha 5-K D$, integrin $\alpha 5$-knockdown-HT1080. ${ }^{\star} P<0.05$; ${ }^{\star \star} P<0.01$; ${ }^{* \star} P<0.001$ compared with Sc using Mann-Whitney $U$-test. Scale bars in a and $\mathbf{d}$ represent $30 \mu \mathrm{m}$. 


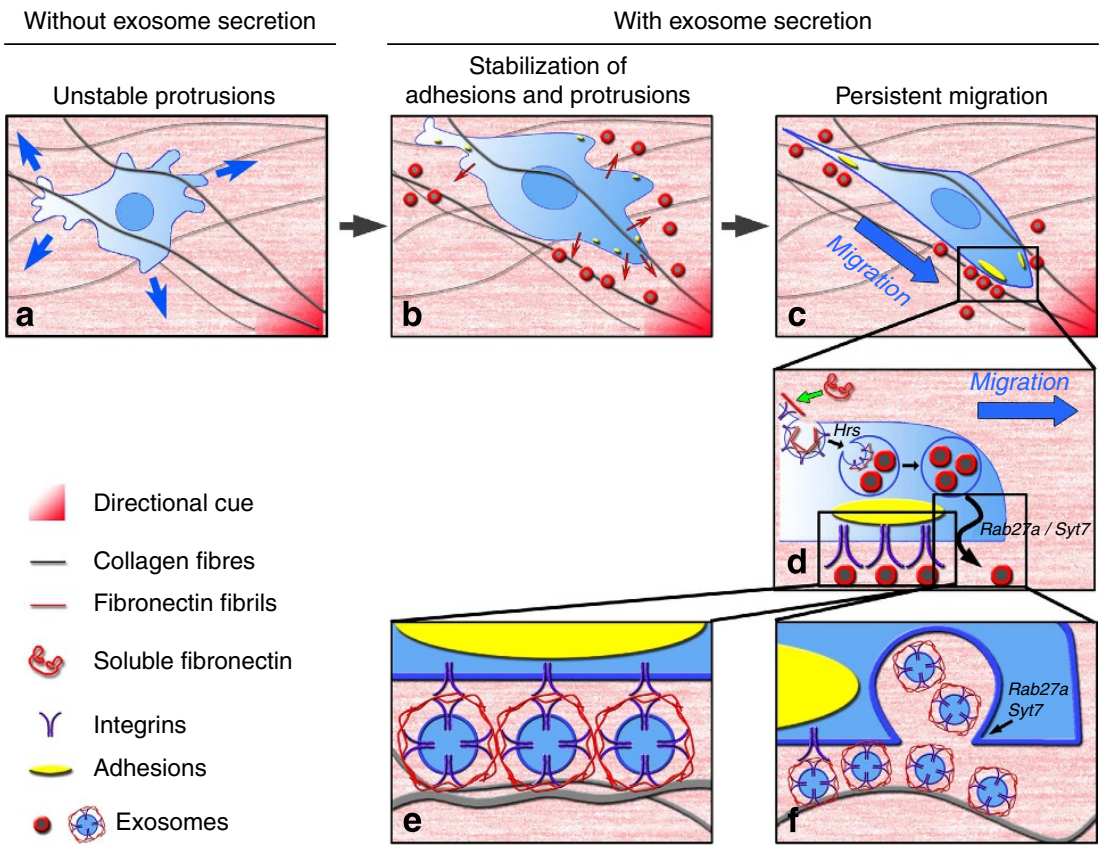

Figure 8 | Proposed model for exosome control of directionally persistent in vivo cell migration (a) In the absence of exosome secretion, cells have unstable protrusions (blue arrows) and are unable to migrate effectively. (b) Secretion of exosomes (yellow) allows cells to effectively respond to directional cues by reinforcing nascent adhesions (orange) and stabilizing protrusions. (c) Positive feedback from exosomes promotes effective and directionally persistent motility, through adhesion enhancement and potentially additional unknown mechanisms. (d) ECM cargoes, such as FN, are carried on exosomes through a process involving endocytosis of ECM-integrin complexes, such as $F N-\alpha 5 \beta 1$, and subsequent sorting into MVEs. (e,f) Autocrine secretion of FN-coated exosomes at the leading edge may allow decoration of collagen fibrils with FN-bound exosomes that can then interact with cellular integrin receptors. Locally elevated concentration of $\mathrm{FN}$-bound exosomes via secretion could facilitate integrin clustering and strong adhesion formation leading to accelerated migration. ECM carried on autocrine-secreted exosomes may be particularly effective at promoting cellular adhesion by matching matrix ligands to the adhesion receptor repertoire of the cell.

formation. Altogether, these data reveal a much more important role for exosome secretion in adhesion formation than previously appreciated $^{36,37}$, and particularly highlight the key role of autocrine exosome secretion in motility processes.

A key question raised by our findings is why does the cell need to place FN on the outside of an exosome to promote adhesion assembly? FN is initially secreted from Golgi-derived vesicles in a soluble non-adhesion-promoting form and then assembled into fibres through interaction with integrins on the cell surface ${ }^{35}$. These fibres may not be optimally positioned to promote migration in one direction and their assembly may not be dynamic enough to respond to fluctuating directional cues. By contrast, endocytosis and resecretion of FN-integrin complexes on exosomes likely facilitates dynamic placement of highly competent adhesive substrata that promote cellular motility. In a previous study, we found that resecretion of fluorescent FN at the bottom of cells occurred within $1 \mathrm{~h}$ of its addition to the culture medium and was rapidly incorporated into nascent streak-like adhesion structures 9 . Presumably this endocytosis-resecretion process is happening continuously so that at any given time there may be a pool of matrix-carrying exosomes in MVE that could be rapidly secreted and used for migration.

$\mathrm{FN}$ is known to be endocytosed in association with integrins and targeted to $\mathrm{MVE}^{8}$ (Fig. $7 \mathrm{~g}$ ). During the intraluminal vesiculation process that creates exosomes within the MVE, the FN would be placed on the outside of the exosome (Supplementary Fig. 2) bound to integrins in the correct topology to interact with both the outside of the cell and with other ECM such as the collagen fibres in the chick CAM (see model, Fig. 8). In vitro, the major source of FN is serum. In the tumour microenvironment, the potential sources of soluble FN are many, including fibroblasts, endothelial cells and tumour cells, as well as leakage from the vasculature. It is also likely that additional matrix molecules could play a similar role, depending on the receptor profile of the migrating cells. In addition, matrix proteolysis could liberate adhesive fragments that could be internalized and then recycled on the outside of exosomes to promote dynamic adhesion formation and motility.

In addition to ECM, exosomes contain numerous cargoes that could impact cell motility in diverse contexts, including proteinases, growth, chemotactic and other extracellular factors $23,26,27,38$. Indeed, our in vivo data indicate that exosomes not only regulate cell speed but also directionality. Because Rab27a-KD cells did not have a defect in persistence in our random motility in vitro assays, we were not able to address exosome control of directionality in that experimental set-up. One possibility is that proteinases could facilitate persistent movement through tissues by remodelling impeding ECM. Another possibility is that exosomes mediate directional movement via chemokines or other factors. Specific identification of which exosome cargoes affect distinct aspects of cell motility is an important future area of research for both the exosome and motility fields. In addition, juxtaposition of multiple motility-promoting factors in a single exosome or closely apposed exosomes could synergistically modulate complex motility behaviours. Understanding these multivariate interactions will be an ongoing challenge for the field.

Use of the pHLuorin-CD63 probe to observe only extracellular CD63 allowed us to achieve a strong signal-to-noise ratio and observe dynamic relationships between exosome secretion and cellular structures. Our observation of adhesive trails left behind migrating cells is reminiscent of slime trails left by lower organisms, including snails, slugs and slime moulds ${ }^{39-41}$. Such slime trails contain complex mixtures of components and serve a 
variety of purposes, including providing adhesive traction without gluing the organism in place ${ }^{39,40}$, and, in the case of slime moulds, as a chemorepellant to prevent recrossing of the same track $^{41}$. We speculate that trails of exosomes from mammalian cells may provide a similar mixture of functions, including not only adhesion but also the ability to modulate other cellular behaviours via diverse cargo molecules.

In this study, we identified a specific cellular organelle, the MVE, as critical to directional cell motility. As polarization is an intrinsic part of directional movement, this finding is consistent with recent findings that targeted MVE secretion is important for formation of the posterior pole of polarized Dictyostelia ${ }^{42}$. In addition, a recent study found that fibroblast-induced exosomes could mobilize Wnt11 from breast cancer cells to induce planar cell polarity signalling 27 . Interestingly, several molecules that have been previously shown to promote directional cell motility are sorted into exosomes and/or regulate their cargo content. Thus, syndecans were recently shown to control exosome biogenesis ${ }^{43}$ and also separately to control directional migration ${ }^{1}$. Likewise, Wnt molecules are major components of exosomes ${ }^{44}$ and together with syndecan- 4 regulate directional cell motility in vivo ${ }^{9,27,45}$. On the basis of these studies and our findings, we speculate that a number of molecules known to control directional cell motility may be doing so via control of exosome biogenesis or cargo content, or as biological cargoes of exosomes.

In summary, we have shown that exosome secretion is essential for directional and efficient cell migration and enhances migration speed by delivering ECM to promote adhesion formation. These data identify a novel feedback pathway that enhances cell motility via local autocrine secretion.

\footnotetext{
Methods

Cell culture and reagents. HT1080 and GFP-expressing HT1080 cells ${ }^{18}$ were maintained in DMEM supplemented with $10 \%$ bovine growth serum (BGS). GFP-expressing HEp3 cells ${ }^{18}$ were maintained in DMEM supplemented with $10 \%$ fetal bovine serum. A lentiviral shRNA expression system, pLKO.1, was used to knockdown Syt7 (TRCN0000007967 (5'-CCGG-CCCTGAATGTCGA GGATAGTA-CTCGAG-TACTATCCTCGACATTCAGGG-TTTTT-3' and TRCN0000007968 (5'-CCGG-GACGATGAAGAGGAACCTGAA-CTCGA G-TTCAGGTTCCTCTTCATCGTC-TTTTT-3'), Thermo Scientific), Rab27a (TRCN0000005296 (5'-CCGG-CGGATCAGTTAAGTGAAGAAA-CTCGAG-TT TCTTCACTTAACTGATCCG-TTTTT-3') and TRCN0000005297 (5'-CCG G-GCTGCCAATGGGACAAACATA-CTCGAG-TATGTTTGTCCCATTGGC AGC-TTTTT- $3^{\prime}$ ), Thermo Scientific), integrin $\alpha 53^{\prime}$ UTR (TRCN0000230126 (5'-CCGG-AGGCAGATCCAGGACTATATT-CTCGAG-AATATAGTCCTGGA TCTGCCT-TTTTTG-3'), Sigma-Aldrich) or scrambled control (Plasmid \#26701 (5'-CCTAAGGTTAAGTCGCCCTCG-CTCGAG-CGAGGGCGACTTAACCTT AGG- $3^{\prime}$ ), Addgene). Hrs shRNAs (5'-GACCTGCTGAAGAGACAAGTC- $3^{\prime}$ and $5^{\prime}$-GCATGAAGAGTAACCACAGC-3') were cloned into pLenti6/Block iT ${ }^{23}$. GFP-paxillin in pRS vector ${ }^{32}$ and mCherry-CD63 in pLenti6 (ref. 23) were used to make stably expressing cell lines. Low-expressing cells were sorted by fluorescenceactivated cell sorting and used for experiments. mCherry-paxillin was a gift from Donna Webb (Vanderbilt University). pHLuorin-CD63 was generated by PCR resulting in the insertion of pHLuorin (a gift from Dr Philippe Chavrier, Institut Curie) between amino acids 42 and 43 in extracellular domain 1 of human CD63 cDNA (a gift from Dr Gillian Griffiths, Cambridge Institute for Medical Research) and recombined into pcDNA3.1. mCherry-Rab5a Q79L was from Addgene. DyLight 550 (62262, Thermo Scientific) was used to label human plasma FN (33016-015, Life Technologies) according to the manufacturer's instructions. GRGESP (44-0-26) and GRGDSP (44-0-24A) peptides were purchased from American Peptide Company. Primary antibodies were: Anti-Syt7 (105 173, Synaptic System, 1:200), anti-Rab27 (R4655, Sigma, 1:1,000), anti-FN (610077, BD Biosciences, 1:5,000), anti-flotillin (610820, BD Biosciences, 1:1,000), anti-Hrs (M-79, Santa Cruz Biotechnology, 1:500), anti-integrin $\alpha 5$ (AB1928, Millipore, 1:1,000), anti-Hsp70 (sc-24, Santa Cruz Biotechnology, 1:2,000), anti-CD63 (ab68418, Abcam, 1:500) and anti- $\beta$-actin (Ac-74, Sigma, 1:5,000). HRP-, Alexa Fluor 680 - or IRDye800- secondary antibodies $(1: 10,000)$ were from Santa Cruz Biotechnology, Invitrogen or Rockland, respectively. Blots were imaged and analysed using a ChemiDoc MP (Bio-Rad) or an Odyssey Infrared Imaging System (Li-Cor Biosciences). Images were cropped for presentation and uncropped blots are presented in Supplementary Fig. 6.
}

The CAM model. Use of the chick CAM to model the formation of metastatic colonies and primary tumours is described in detail elsewhere ${ }^{17,18}$. In brief, fertilized eggs decanted into sterilized weigh boats were allowed to mature ex ovo for 9 days. GFP-expressing cells were injected either i.v. or within the CAM tissue on developmental day 10. At indicated times, the ex ovo chick embryos were placed in a custom intravital imaging chamber ${ }^{46,47}$. Images of tumour cells in the CAM were acquired using a Lumar V12 fluorescence stereomicroscope (Zeiss) or an Olympus BX61 equipped with a digital camera controlled with Volocity image acquisition software (PerkinElmer).

In vivo analysis of cell migration and protrusion dynamics. GFP-expressing HT1080 cells $\left(1 \times 10^{5}\right)$ were injected i.v. into the CAM of ex ovo chicks on developmental day 10 . At $24 \mathrm{~h}$ post injection, the ex ovo chicks were transferred to an intravital imaging chamber and placed under a fully automated temperaturecontrolled microscope $\left(99^{\circ} \mathrm{F}, \mathrm{BX} 61\right.$, Olympus $\left.{ }^{18}\right)$. Time-lapse migration movies were captured every $15 \mathrm{~min}$ for $16 \mathrm{~h}$ using $\times 10 / 0.40$ UPlanSApo objective lens, whereas protrusion dynamics movies were captured at every $2 \mathrm{~min}$ for $1 \mathrm{~h}$ using $\times 20 / 0.70 \mathrm{~W}$ UApo/340 water objective lens. To analyse in vivo cell migration, the nuclear position of each cell was tracked and cell speed, displacement rate and persistence index were analysed using Volocity. For protrusion dynamics analysis, the aspect ratio was calculated using Fiji, the number of protrusions formed was counted manually and length and persistence of protrusions were measured using Metamorph software (Molecular Devices).

Isolation of exosomes. To collect conditioned media, $80 \%$ confluent cells were cultured for $48 \mathrm{~h}$ in Opti-MEM. Exosomes were isolated from conditioned media by serial centrifugation at $300 \mathrm{~g}$ for $10 \mathrm{~min}, 2,000 \mathrm{~g}$ (4,000 r.p.m. in Ti45 rotor) for $30 \mathrm{~min}, 10000 \mathrm{~g}$ for $30 \mathrm{~min}(9,300$ r.p.m. in Ti45) and 100,000 (30,000 r.p.m. in Ti45) for overnight to, respectively, sediment live cells, dead cells, debris and microvesicles and exosomes $\left(\sim 3.5 \mu \mathrm{g}\right.$ exosomes from $\left.1 \times 10^{6} \mathrm{HT} 1080 \mathrm{~s}\right)$. For further purification of UC-exosomes, the discontinuous iodixanol gradient was prepared $^{48}$. Solutions $(40 \%(\mathrm{w} / \mathrm{v}), 20 \%(\mathrm{w} / \mathrm{v}), 10 \%(\mathrm{w} / \mathrm{v})$ and $5 \%(\mathrm{w} / \mathrm{v}))$ of iodixanol were made by diluting OptiPrep (60\% (w/v) aqueous iodixanol, Axis-Shield PoC) with $0.25 \mathrm{M}$ sucrose $/ 10 \mathrm{mM}$ Tris, $\mathrm{pH} 7.5$ from the bottom to the top of a $14 \times 89 \mathrm{~mm}$ polyallomer tube. UC-exosomes were added on a top of the gradient and continuous gradient was made through UC at 100,000g (24,000 r.p.m. in SW40 Ti rotor) for $18 \mathrm{~h}$. Twelve fractions were collected and each fraction was diluted in PBS. Each fraction was pelleted through another round of ultracentrifugation at $100,000 \mathrm{~g}$ for $3 \mathrm{~h}$, washed with PBS and resuspended in PBS. To deplete FN in exosomes, cells were cultured in DMEM supplemented with 10\% FN-depleted $\mathrm{BGS}^{9}$ for 10 days before collecting conditioned medium for exosome isolation. Exosomes were quantitated by NanoSight tracking analysis.

In vitro single-cell migration assay. For coating experiments, tissue culture 24-well plates were incubated with appropriately exosome or human plasma FN (Gibco BRL) suspensions in PBS at $4^{\circ} \mathrm{C}$ overnight before washing and using. Trypsinized cells (3,000 in each well) were then cultured overnight on the coated plates before capturing and analysing time-lapse migration movies using a Nikon Eclipse TE2000E microscope equipped with a $37^{\circ} \mathrm{C}$ chamber as previously described ${ }^{9}$. The nuclear position of each cell was tracked and cell speed and persistence index was calculated using Metamorph software. For in vitro migration with addback of exosomes in the media, cells were allowed to adhere to tissue culture well plates for $5 \mathrm{~h}$ before changing the media to DMEM supplemented with $10 \%$ exosome-depleted BGS (prepared by ultracentrifugation for $12 \mathrm{~h}$ at $100,000 \mathrm{~g}$ ) \pm purified exosomes. Then, live-cell imaging was performed as described above.

Adhesion assembly and disassembly assay. GFP-paxillin-expressing cells were plated on glass-bottom dishes (MatTek) coated with $1 \mu \mathrm{g} \mathrm{ml}^{-1} \mathrm{FN}$ and allowed to adhere for $1 \mathrm{~h}$. Images were acquired with an LSM 510 laser scanning confocal microscope (CarlZeiss) equipped with a $\times 63 / 1.40$ NA Plan Apo oil objective lens at $37^{\circ} \mathrm{C}$ with $5 \% \mathrm{CO}_{2}$. Images were taken every $30 \mathrm{~s}$ for $45 \mathrm{~min}$. The rate constants for assembly and disassembly of adhesions were measured through the Focal Adhesion Analysis Server ${ }^{49}$.

Live imaging of CD63 and focal adhesions. Either mCherry-CD63 was stably expressed in stable GFP-paxillin-expressing HT1080 or pHLuorin-CD63 was transiently co-transfected into HT1080 along with mCherry-paxillin or mCherryRab5A Q79L. A day after transfection, cells were replated on glass-bottom dishes coated with FN $\left(1 \mu \mathrm{g} \mathrm{ml}^{-1}\right)$. Images of mCherry-CD63/GFP-paxillin- or mCherry-Rab5A Q79L/pHLuorin-CD63-expressing cells were acquired with LSM 710 META laser scanning confocal microscope equipped with a $\times 63 / 1.40$ PlanApochromat oil objective lens at $37^{\circ} \mathrm{C}$ with $5 \% \mathrm{CO}_{2}$. Epifluorescence images of pHLuorin-CD63-expressing cells were acquired with Nikon Eclipse TE2000E microscope equipped with a $37^{\circ} \mathrm{C}$ chamber using a $\times 40 / 0.60$ Plan Fluor lens. For migration movies, images were taken every 2 min for $4 \mathrm{~h}$. For lamellipodial protrusion analysis by kymography, images were taken every $6 \mathrm{~s}$ for $20 \mathrm{~min}$. Images of pHLuorin-CD63/mCherry-paxillin-expressing cells were taken with a Nikon Eclipse TiE TIRF microscope equipped with a Perfect Focus System using a TIRF 
$\times$ 100/1.49 NA oil-immersion lens and Neo 5.5 cMOS camera (Andor). For each cell, images were taken every $30 \mathrm{~s}$ for $45 \mathrm{~min}$, yielding 91 frames per movie.

Numbers and statistics. For both quantitated data and representative images from experiments, the $n$ values and independent experiment numbers are listed in the figure legends. Imaging data were acquired from at least three independent experiments and multiple images or movies on multiple cells were captured. Cell numbers to be quantitated for motility experiments were determined by our experience and analyses were excluded only if there is an obvious reason for poor data, such as dead or sick-looking cells. For non-quantitated western blots (for example, checking knockdown), they were generally performed a single time. All data sets were tested for normality using the Kolmogorov-Smirnov normality test in SPSS software (IBM). Non-parametric data groups were compared using the Mann-Whitney $U$-test and plotted as box and whiskers, in which the box indicates the 25-75th percentile and the whiskers indicate the 5-95th percentile, with the line indicating the median. Parametric data were compared using Student's $t$-test and plotted as mean \pm s.e. in bar graphs.

\section{References}

1. Petrie, R. J., Doyle, A. D. \& Yamada, K. M. Random versus directionally persistent cell migration. Nat. Rev. Mol. Cell Biol. 10, 538-549 (2009).

2. Millarte, V. \& Farhan, H. The Golgi in cell migration: regulation by signal transduction and its implications for cancer cell metastasis. TheScientificWorldJournal 2012, 498278 (2012).

3. Miller, P. M. et al. Golgi-derived CLASP-dependent microtubules control Golgi organization and polarized trafficking in motile cells. Nat. Cell Biol. 11, 1069-1080 (2009).

4. Prigozhina, N. L. \& Waterman-Storer, C. M. Protein kinase D-mediated anterograde membrane trafficking is required for fibroblast motility. Curr. Biol. 14, 88-98 (2004).

5. Caswell, P. T., Vadrevu, S. \& Norman, J. C. Integrins: masters and slaves of endocytic transport. Nat. Rev. Mol. Cell Biol. 10, 843-853 (2009).

6. Margadant, C., Monsuur, H. N., Norman, J. C. \& Sonnenberg, A. Mechanisms of integrin activation and trafficking. Curr. Opin. Cell Biol. 23, 607-614 (2011).

7. Dozynkiewicz, M. A. et al. Rab25 and CLIC3 collaborate to promote integrin recycling from late endosomes/lysosomes and drive cancer progression. Dev. Cell 22, 131-145 (2012).

8. Lobert, V. H. et al. Ubiquitination of alpha 5 beta 1 integrin controls fibroblast migration through lysosomal degradation of fibronectin-integrin complexes. Dev. Cell 19, 148-159 (2010).

9. Sung, B. H., Zhu, X., Kaverina, I. \& Weaver, A. M. Cortactin controls cell motility and lamellipodial dynamics by regulating ECM secretion. Curr. Biol. 21, 1460-1469 (2011).

10. Benesh, E. C. et al. Bves and NDRG4 regulate directional epicardial cell migration through autocrine extracellular matrix deposition. Mol. Biol. Cell. 24, 3496-3510 (2013).

11. Colvin, R. A. et al. Synaptotagmin-mediated vesicle fusion regulates cell migration. Nat. Immunol. 11, 495-502 (2010).

12. Proux-Gillardeaux, V., Raposo, G., Irinopoulou, T. \& Galli, T. Expression of the Longin domain of TI-VAMP impairs lysosomal secretion and epithelial cell migration. Biol. Cell 99, 261-271 (2007)

13. Thery, C. Exosomes: secreted vesicles and intercellular communications. F1000 Biol. Rep. 3, 15 (2011).

14. Koumangoye, R. B., Sakwe, A. M., Goodwin, J. S., Patel, T. \& Ochieng, J. Detachment of breast tumor cells induces rapid secretion of exosomes which subsequently mediate cellular adhesion and spreading. PLoS ONE 6, e24234 (2011).

15. Mu, W., Rana, S. \& Zoller, M. Host matrix modulation by tumor exosomes promotes motility and invasiveness. Neoplasia 15, 875-887 (2013).

16. Lewis, J. D. et al. Viral nanoparticles as tools for intravital vascular imaging. Nat. Med. 12, 354-360 (2006).

17. Palmer, T. D., Lewis, J. \& Zijlstra, A. Quantitative analysis of cancer metastasis using an avian embryo model. J. Vis. Exp. 51, e2815 doi:10.3791/2815 (2011).

18. Zijlstra, A., Lewis, J., Degryse, B., Stuhlmann, H. \& Quigley, J. P. The inhibition of tumor cell intravasation and subsequent metastasis via regulation of in vivo tumor cell motility by the tetraspanin CD151. Cancer Cell 13, 221-234 (2008).

19. Borm, B., Requardt, R. P., Herzog, V. \& Kirfel, G. Membrane ruffles in cell migration: indicators of inefficient lamellipodia adhesion and compartments of actin filament reorganization. Exp. Cell Res. 302, 83-95 (2005).

20. Goldfinger, L. E., Han, J., Kiosses, W. B., Howe, A. K. \& Ginsberg, M. H. Spatial restriction of alpha4 integrin phosphorylation regulates lamellipodial stability and alpha4beta1-dependent cell migration. J. Cell Biol. 162, 731-741 (2003).

21. Owen, K. A. et al. Regulation of lamellipodial persistence, adhesion turnover, and motility in macrophages by focal adhesion kinase. J. Cell Biol. 179, 1275-1287 (2007)

22. Ostrowski, M. et al. Rab27a and Rab27b control different steps of the exosome secretion pathway. Nat. Cell Biol. 12, 19-30 sup pp 11-13 (2010).
23. Hoshino, D. et al. Exosome secretion is enhanced by invadopodia and drives invasive behavior. Cell Rep. 5, 1159-1168 (2013).

24. Martinez, I. et al. Synaptotagmin VII regulates $\mathrm{Ca}(2+)$-dependent exocytosis of lysosomes in fibroblasts. J. Cell Biol. 148, 1141-1149 (2000).

25. Palmer, T. D. et al. Integrin-free tetraspanin CD151 can inhibit tumor cell motility upon clustering and is a clinical indicator of prostate cancer progression. Cancer Res. 74, 173-187 (2014).

26. Higginbotham, J. N. et al. Amphiregulin exosomes increase cancer cell invasion. Curr. Biol. 21, 779-786 (2011).

27. Luga, V. et al. Exosomes mediate stromal mobilization of autocrine Wnt-PCP signaling in breast cancer cell migration. Cell 151, 1542-1556 (2012)

28. Hanson, P. I. \& Cashikar, A. Multivesicular body morphogenesis. Annu. Rev. Cell Dev. Biol. 28, 337-362 (2012).

29. Tamai, K. et al. Exosome secretion of dendritic cells is regulated by Hrs, an ESCRT-0 protein. Biochem. Biophys. Res. Commun. 399, 384-390 (2010).

30. Miesenbock, G., De Angelis, D. A. \& Rothman, J. E. Visualizing secretion and synaptic transmission with $\mathrm{pH}$-sensitive green fluorescent proteins. Nature 394, 192-195 (1998)

31. Sun, Q., Westphal, W., Wong, K. N., Tan, I. \& Zhong, Q. Rubicon controls endosome maturation as a Rab7 effector. Proc. Natl Acad. Sci. USA 107, 19338-19343 (2010).

32. Bryce, N. S. et al. Cortactin promotes cell motility by enhancing lamellipodial persistence. Curr. Biol. 15, 1276-1285 (2005).

33. Huttenlocher, A. \& Horwitz, A. R. Integrins in cell migration. Cold Spring Harb. Perspect. Biol. 3, a005074 (2011).

34. Nayal, A. et al. Paxillin phosphorylation at Ser273 localizes a GIT1-PIX-PAK complex and regulates adhesion and protrusion dynamics. J. Cell Biol. 173, 587-589 (2006)

35. Singh, P., Carraher, C. \& Schwarzbauer, J. E. Assembly of fibronectin extracellular matrix. Annu. Rev. Cell Dev. Biol. 26, 397-419 (2010).

36. Clayton, A. et al. Adhesion and signaling by B cell-derived exosomes: the role of integrins. FASEB J. 18, 977-979 (2004).

37. Deng, Z. et al. Tumor cell cross talk with tumor-associated leukocytes leads to induction of tumor exosomal fibronectin and promotes tumor progression. Am. J. Pathol. 180, 390-398 (2012).

38. Chen, T., Guo, J., Yang, M., Zhu, X. \& Cao, X. Chemokine-containing exosomes are released from heat-stressed tumor cells via lipid raft-dependent pathway and act as efficient tumor vaccine. J. Immunol. 186, 2219-2228 (2011)

39. Denny, M. Locomotion: the cost of gastropod crawling. Science 208, 1288-1290 (1980).

40. Ewoldt, R. H., Clasen, C., Hosoi, A. E. \& McKinley, G. H. Rheological fingerprinting of gastropod pedal mucus and synthetic complex fluids for biomimicking adhesive locomotion. Soft Matter 3, 634-643 (2007).

41. Reid, C. R., Latty, T., Dussutour, A. \& Beekman, M. Slime mold uses an externalized spatial "memory" to navigate in complex environments. Proc. Natl Acad. Sci. USA 109, 17490-17494 (2012).

42. Shen, B., Fang, Y., Wu, N. \& Gould, S. J. Biogenesis of the posterior pole is mediated by the exosome/microvesicle protein-sorting pathway. J. Biol. Chem. 286, 44162-44176 (2011).

43. Baietti, M. F. et al. Syndecan-syntenin-ALIX regulates the biogenesis of exosomes. Nat. Cell Biol. 14, 677-685 (2012).

44. Gross, J. C., Chaudhary, V., Bartscherer, K. \& Boutros, M. Active Wnt proteins are secreted on exosomes. Nat. Cell Biol. 14, 1036-1045 (2012).

45. Matthews, H. K. et al. Directional migration of neural crest cells in vivo is regulated by Syndecan-4/Racl and non-canonical Wnt signaling/RhoA. Development 135, 1771-1780 (2008).

46. Cho, C. F., Ablack, A., Leong, H. S., Zijlstra, A. \& Lewis, J. Evaluation of nanoparticle uptake in tumors in real time using intravital imaging. J. Vis. Exp. 52, e2808 doi:10.3791/2808 (2011).

47. Kain, K. H. et al. The chick embryo as an expanding experimental model for cancer and cardiovascular research. Dev. Dyn. 243, 216-228 (2014).

48. Tauro, B. J. et al. Comparison of ultracentrifugation, density gradient separation, and immunoaffinity capture methods for isolating human colon cancer cell line LIM1863-derived exosomes. Methods 56, 293-304 (2012).

49. Berginski, M. E. \& Gomez, S. M. The Focal Adhesion Analysis Server: a web tool for analyzing focal adhesion dynamics. F1000Research 2, 68 (2013).

\section{Acknowledgements}

This study was funded by NCI grants R01 CA163592, U01CA143069, U19 CA179514 to A.M.W. and CA143081 to A.Z., by American Cancer Society RSG-09-170-01-CSM to A.M.W. and American Heart Association Research Grant-in-Aid 13GRNT16580020 to A.M.W. The Vanderbilt Cell Imaging Shared Resource (CISR) and/or Flow Cytometry facilities are supported by grants CA68485, DK20593, DK58404, HD15052, DK59637 and EY08126. We thank Irina Kaverina and Matt Tyska for use of their TIRF microscopes and Robert Coffey for use of NanoSight. The content of this article is solely the responsibility of the authors and does not necessarily represent the official views of the National Institutes of Health. In addition, we are grateful to Tyson Foods Inc. for generously providing the chick embryos for these studies. 


\section{Author contributions}

B.H.S., A.Z. and A.M.W. designed the study, analysed the data and wrote the manuscript. B.H.S. performed most experiments and analyses. T.K. injected cells in the CAM and recorded in vivo movies. D.H. designed and cloned the pHLuorin-CD63 construct and performed validation experiments in Supplementary Figure 5 .

\section{Additional information}

Supplementary Information accompanies this paper at http://www.nature.com/ naturecommunications

Competing financial interests: The authors declare no competing financial interests.
Reprints and permission information is available online at http://npg.nature.com/ reprintsandpermissions/

How to cite this article: Sung, B.H. et al. Directional cell movement through tissues is controlled by exosome secretion. Nat. Commun. 6:7164 doi: 10.1038/ncomms8164 (2015).

(c) (i) This work is licensed under a Creative Commons Attribution 4.0 International License. The images or other third party material in this article are included in the article's Creative Commons license, unless indicated otherwise in the credit line; if the material is not included under the Creative Commons license, users will need to obtain permission from the license holder to reproduce the material. To view a copy of this license, visit http://creativecommons.org/licenses/by/4.0/ 